\title{
Trends in Medicinal Uses of Edible Wild Vertebrates in Brazil
}

\author{
Rômulo Romeu Nóbrega Alves, ${ }^{1}$ Tacyana Pereira Ribeiro Oliveira, ${ }^{2}$ \\ and Maria Franco Trindade Medeiros ${ }^{3}$ \\ ${ }^{1}$ Departamento de Biologia, Universidade Estadual da Paraíba, 58429-500 Campina Grande, PB, Brazil \\ ${ }^{2}$ Centro de Ciências Biológicas e Sociais Aplicadas (CCBSA), Universidade Estadual da Paraíba, 58071-160 João Pessoa, PB, Brazil \\ ${ }^{3}$ Unidade Acadêmica de Biologia e Química, Universidade Federal de Campina Grande, 58175-000 Cuité, PB, Brazil
}

Correspondence should be addressed to Maria Franco Trindade Medeiros; mariaftm@hotmail.com

Received 5 April 2017; Accepted 3 July 2017; Published 15 August 2017

Academic Editor: Ghee T. Tan

Copyright (c) 2017 Rômulo Romeu Nóbrega Alves et al. This is an open access article distributed under the Creative Commons Attribution License, which permits unrestricted use, distribution, and reproduction in any medium, provided the original work is properly cited.

\begin{abstract}
The use of food medicines is a widespread practice worldwide. In Brazil, such use is often associated with wild animals, mostly focusing on vertebrate species. Here we assessed taxonomic and ecological trends in traditional uses of wild edible vertebrates in the country, through an extensive ethnobiological database analysis. Our results showed that at least 165 health conditions are reportedly treated by edible vertebrate species $(n=204)$, mostly fishes and mammals. However, reptiles stand out presenting a higher plasticity in the treatment of multiple health conditions. Considering the 20 disease categories recorded, treatment prescriptions were similar within continental (i.e., terrestrial and freshwater) and also within coastal and marine habitats, which may reflect locally related trends in occurrence and use of the medicinal fauna. The comprehension of the multiplicity and trends in the therapeutic uses of Brazilian vertebrates is of particular interest from a conservation perspective, as several threatened species were recorded.
\end{abstract}

\section{Introduction}

Wildlife represents an immeasurable source of raw materials that support health systems of different human cultures that depend on nature as a source of medicines to treat and cure illnesses [1]. Plants and animals have been used as medicinal sources since ancient times, and even today animal- and plant-based pharmacopeias continue to play an essential role in health care. Although plants and plant-derived materials make up the majority of the ingredients used in most traditional medical systems globally, whole animals, animal parts, and animal-derived products also constitute important elements of the materia medica [2-6].

The use of animal species as remedies, although representing an important component of traditional medicines (sometimes in association with plant species), has been much less studied than medicinal plants [1]. However, the importance of nonbotanical remedies (those of animal and mineral origin) is emerging [7], resulting in a recent boom in publications focusing on zootherapy [8-11].
Brazil is well known for its rich social/cultural diversity, as represented by more than two hundred indigenous people and a range of local communities, which in turn have contributed to the high diversity of traditional knowledge and practices which include the use of medicinal animals. Indeed, animals have been used as a source of medicine in the country and have played a significant role in healing practices as many people have used animals as medicines or alternative or supplementary treatments $[12,13]$.

Hence, Brazil can be considered a model to extensive zootherapeutic studies, since the use of animals and animalderived products is widespread among the country's human cultures, as predicted by the zootherapeutic universality hypothesis [14]. Furthermore, the concomitant use of wild animals for nutritional and medicinal purposes is also diffuse in several localities in the country, thus highlighting their important role as food medicine in well-established folk medical practices [15].

Recent research has highlighted the predominant use of vertebrates as medicinal fauna in different medical systems 
worldwide [1]. As remarked by Perry [16], this is an expected trend, considering the frequent interactions between people and vertebrates-typically large-bodied animals, which may provide a wide range of medicinal products. This raises particular conservation concerns, as some of these taxa are overharvested for their medicinal uses and are now threatened [1].

In this article, we provide an assessment of the uses of wild edible vertebrate species in Brazilian Traditional Medicine. The study focused on the following questions: (1) which edible vertebrate taxa are mostly used in the Brazilian Traditional Medicine? (2) Do the conditions treated by medicinal resources vary with taxonomical group and/or animal's habitat?

\section{Methods}

Data used in this research resulted from an extensive analysis of the ethnozoological database provided by the Laboratório de Etnozoologia, Universidade Estadual da Paraíba. The database comprises information from ethnozoological studies on faunal medicinal use performed in all Brazilian regions. Additional data was gathered through information available in reviews published by the laboratory researchers [17-19].

Data analysis comprised information on species of edible vertebrates used as medicines, their family classification, habitats, conservation status, and conditions to which animals were prescribed. We only considered those taxa that could be identified to species level, and the scientific nomenclature of the taxa recorded (fishes, amphibians, reptiles, and mammals) and/or habitats were in accordance with the following databases: Fishbase (Froese and Pauly, 2016; http://www.fishbase.org/), Amphibian Species of the World (http://research.amnh.org/herpetology/amphibia/ index.php), The Reptile Database (http://www.reptiledatabase.org/), and Mammal Species of the World [20]. With regard to habitat analysis, marine and estuarine species were grouped in the same category (i.e., coastal and marine); if a marine species was also reported to freshwater environments, it habitat was categorized as costal and marine/freshwater. Moreover, continental species which could inhabit both terrestrial and aquatic systems were considered as semiaquatic species.

The conservation status of the analysed species follows the International Union for the Conservation of Nature [21], the Convention on International Trade in Endangered Species of Wild Fauna and Flora [22], and the Brazilian red lists (decrees 444 and 445, Brazilian Ministry of Environment, 2014). Health conditions considered in this research were categorized by the International Statistical Classification of Diseases and Related Health Problems (ICD-10 Version: 2016; http://apps.who.int/classifications/icd10/browse/2016/en).

2.1. Data Analysis. All data were verified for normal distribution (Shapiro-Wilk's test) and homogeneity of variance (Levene's test) and nonparametric tests were performed when those assumptions were not met.

A Kruskal-Wallis test (followed by Dunn's post hoc test) and an ANOVA were performed to determine whether the

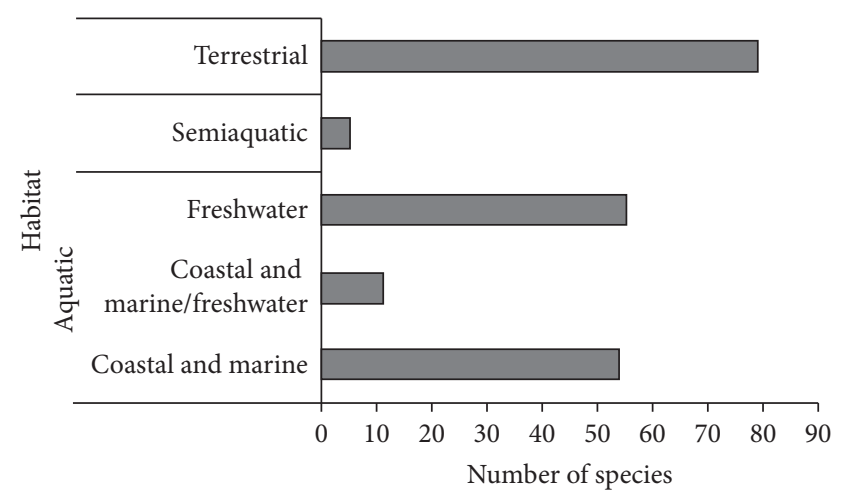

Figure 1: Number of edible vertebrates used in traditional medicines in Brazil, according to their habitat types.

number of health conditions treated per species varied among vertebrate taxonomic groups or habitat types, respectively. Resemblance between health conditions treated (grouped into ICD's categories) and taxonomic groups or animals' habitat types were assessed based on Jaccard's similarity index, where resulting matrices were used to perform cluster analyses. Due to low number of species recorded $(n=3)$, amphibians were excluded from all statistical analysis regarding taxonomic groups.

\section{Results}

At least 204 edible vertebrate species have been used in Brazilian Traditional Medicine (see Table 1). Fishes were the most represented group ( $n=97$ species), followed by mammals $(n=48)$, reptiles $(n=29)$, birds $(n=27)$, and amphibians $(n=3)$. Most medicinal animals are aquatic (58.9\%), mostly inhabiting freshwater (27.0\% of total counts) and coastal/marine $(26.5 \%$ of total counts) environments (Figure 1). Terrestrial and semiaquatic vertebrates corresponded to $38.7 \%$ and $2.5 \%$ of medicinal vertebrates recorded, respectively.

Edible medicinal vertebrates were reportedly used to treat 165 health conditions/diseases (see Table 2). A single illness could be treated by various animal species (e.g., 67 animal species were used in the treatment of asthma and 60 in the treatment of rheumatism), and although most species (particularly fishes, mammals, and birds) were used to treat only one $(n=85 ; 41.7 \%)$ or up to five illnesses $(n=156$; $76.5 \%$ ), several were prescribed for treating multiple illnesses ( $>5$ conditions; $n=48,23.5 \%$ ), as shown in Figure 2. Reptiles were the most versatile group, as they were mostly used in the treatment of multiple conditions, with almost half of the species $(n=14)$ being used to treat more than 10 illnesses (Figure 2). Indeed, from the 10 most expressive species in the treatment of multiple conditions (see Table 1), seven are reptiles, for instance, the "teju" and the boa snake (Salvator teguixin and Boa constrictor, resp.; $n=28$ health conditions prescribed, each), the Neotropical rattlesnake (Crotalus durissus; $n=27$ conditions), the green sea turtle (Chelonia mydas; $n=25$ conditions), and the common caiman (Caiman crocodilus; $n=24$ conditions). Moreover, 
TABLE 1: Vertebrate species (scientific and common names, when available) used as medicine food in Brazil, with remarks to habitats, conservation status, and health conditions to which each animal is prescribed. Families and species name in alphabetical order.

\begin{tabular}{|c|c|c|}
\hline Taxa & Health conditions treated & Habitat \\
\hline \multicolumn{3}{|l|}{ Fishes } \\
\hline \multicolumn{3}{|l|}{ Anostomidae } \\
\hline $\begin{array}{l}\text { Leporinus friderici (Bloch, 1794), } \\
\text { threespot leporinus }\end{array}$ & Earache & Freshwater \\
\hline $\begin{array}{l}\text { Schizodon knerii (Steindachner, } \\
\text { 1875), "piau-branco" }\end{array}$ & Leucoma, edema & Freshwater \\
\hline \multicolumn{3}{|l|}{ Arapaimidae } \\
\hline $\begin{array}{l}\text { Arapaima gigas (Schinz, 1822), } \\
\text { "arapaima," "pirarucu," "pirosca" }\end{array}$ & Asthma, pneumonia & Freshwater \\
\hline \multicolumn{3}{|l|}{ Arhynchobatidae } \\
\hline $\begin{array}{l}\text { Atlantoraja cyclophora (Regan, } \\
\text { 1903), eyespot skate }\end{array}$ & Haemorrhage after delivery & Marine \\
\hline \multicolumn{3}{|l|}{ Ariidae } \\
\hline $\begin{array}{l}\text { Aspistor luniscutis (Valenciennes, } \\
\text { 1840), "bagre-amarelo" }\end{array}$ & $\begin{array}{l}\text { Pain relief in injuries caused by } \\
\text { the species' sting }\end{array}$ & Marine/brackish \\
\hline $\begin{array}{l}\text { Bagre bagre (Linnaeus, 1766), coco } \\
\text { sea catfish, "bagre-fidalgo" }\end{array}$ & Injuries caused by itself & Marine/brackish \\
\hline $\begin{array}{l}\text { Genidens barbus (Lacepède, 1803), } \\
\text { white sea catfish, "bagre-do-mangue" }\end{array}$ & $\begin{array}{l}\text { Pain relief in injuries caused by } \\
\text { the species' sting }\end{array}$ & Marine/brackish \\
\hline $\begin{array}{l}\text { Genidens genidens (Cuvier, 1829), } \\
\text { sea catfish, "bagre" }\end{array}$ & Injuries caused by itself & Marine/brackish \\
\hline \multicolumn{3}{|l|}{ Aspredinidae } \\
\hline $\begin{array}{l}\text { Aspredinichthys tibicen } \\
\text { (Valenciennes, 1840), tenbarbed banjo, } \\
\text { "viola" }\end{array}$ & Asthma & Freshwater/brackish \\
\hline $\begin{array}{l}\text { Aspredo aspredo (Linnaeus, 1758), } \\
\text { banjo catfish, "banjo," "viola" }\end{array}$ & Asthma & Freshwater/brackish \\
\hline \multicolumn{3}{|l|}{ Auchenipteridae } \\
\hline $\begin{array}{l}\text { Trachelyopterus galeatus (Linnaeus, } \\
\text { 1766),“cumbá” }\end{array}$ & $\begin{array}{l}\text { Umbilical hernia, asthma, sexual } \\
\text { impotence }\end{array}$ & Freshwater \\
\hline \multicolumn{3}{|l|}{ Balistidae } \\
\hline $\begin{array}{l}\text { Balistes capriscus Gmelin, 1789, grey } \\
\text { triggerfish, "peixe-porco" }\end{array}$ & Bronchitis & Marine/reef \\
\hline $\begin{array}{l}\text { Balistes vetula Linnaeus, 1758, queen } \\
\text { triggerfish, "cangulo," "capado," “peroá" }\end{array}$ & $\begin{array}{l}\text { Stroke, asthma, thrombosis, } \\
\text { earache, pain relief in injuries } \\
\text { caused by the species' sting, } \\
\text { haemorrhage, ascites, } \\
\text { schistosomiasis, appendicitis, } \\
\text { menstrual cramps, gastritis }\end{array}$ & Marine/reef \\
\hline \multicolumn{3}{|l|}{ Batrachoididae } \\
\hline $\begin{array}{l}\text { Thalassophryne nattereri } \\
\text { Steindachner, 1876, venomous toadfish, } \\
\text { "niquim" }\end{array}$ & $\begin{array}{l}\text { Pain relief caused in injuries by } \\
\text { the species' sting }\end{array}$ & Marine/brackish \\
\hline \multicolumn{3}{|l|}{ Bryconidae } \\
\hline $\begin{array}{l}\text { Brycon nattereri Günther, } 1864, \\
\text { "pirapitinga," "matrinchã" }\end{array}$ & Flu & Freshwater \\
\hline \multicolumn{3}{|l|}{ Callichthyidae } \\
\hline $\begin{array}{l}\text { Callichthys callichthys (Linnaeus, } \\
\text { 1758), "cascarudo," "caboge" }\end{array}$ & Asthma, umbilical hernia & Freshwater \\
\hline \multicolumn{3}{|l|}{ Lamnidae } \\
\hline $\begin{array}{l}\text { Carcharodon carcharias (Linnaeus, } \\
\text { 1758), great white shark }\end{array}$ & Osteoporosis & Marine/brackish \\
\hline $\begin{array}{l}\text { Isurus oxyrinchus Rafinesque, 1810, } \\
\text { shortfin mako }\end{array}$ & Osteoporosis & Marine \\
\hline
\end{tabular}


TABle 1: Continued.

\begin{tabular}{|c|c|c|}
\hline Taxa & Health conditions treated & Habitat \\
\hline \multicolumn{3}{|l|}{ Carcharhinidae } \\
\hline $\begin{array}{l}\text { Carcharhinus falciformis (Müller \& } \\
\text { Henle, 1839), silky shark }\end{array}$ & Osteoporosis & Marine/reef \\
\hline $\begin{array}{l}\text { Carcharhinus leucas (Müller \& } \\
\text { Henle, 1839), bull shark, } \\
\text { "tubarão-cabeça-chata" }\end{array}$ & Osteoporosis & Marine/brackish/reef/freshwater \\
\hline $\begin{array}{l}\text { Carcharhinus limbatus (Müller \& } \\
\text { Henle, 1839), blacktip shark, "sucuri } \\
\text { preto" }\end{array}$ & Osteoporosis & Marine/brackish/reef \\
\hline $\begin{array}{l}\text { Carcharhinus obscurus (Lesueur, } \\
\text { 1818), dusky shark }\end{array}$ & Osteoporosis & Marine/brackish/reef \\
\hline $\begin{array}{l}\text { Carcharhinus porosus (Ranzani, } \\
\text { 1839), smalltail shark, "junteiro," } \\
\text { "cação-galha-preta”" }\end{array}$ & $\begin{array}{l}\text { Asthma, rheumatism, wounds, } \\
\text { inflammations, osteoporosis, } \\
\text { anaemia }\end{array}$ & Marine/brackish \\
\hline $\begin{array}{l}\text { Galeocerdo cuvier (Péron \& Lesueur, } \\
\text { 1822), tiger shark, "jaguara" }\end{array}$ & Osteoporosis & Marine/brackish/reef \\
\hline $\begin{array}{l}\text { Negaprion brevirostris (Poey, 1868), } \\
\text { lemon shark }\end{array}$ & Osteoporosis & Marine/brackish/reef \\
\hline $\begin{array}{l}\text { Rhizoprionodon lalandii (Müller \& } \\
\text { Henle, 1839), Brazilian sharpnose shark, } \\
\text { "cação" }\end{array}$ & Rheumatism, osteoporosis & Marine \\
\hline $\begin{array}{l}\text { Rhizoprionodon porosus (Poey, 1861), } \\
\text { Caribbean sharpnose shark, "cação" }\end{array}$ & Rheumatism, osteoporosis & Marine/brackish/reef/freshwater \\
\hline \multicolumn{3}{|l|}{ Sphyrnidae } \\
\hline $\begin{array}{l}\text { Sphyrna lewini (Griffith \& Smith, } \\
\text { 1834), scalloped hammerhead, } \\
\text { "peixe-martelo," “cação-panã," } \\
\text { "cação-chapéu” }\end{array}$ & $\begin{array}{l}\text { Asthma, wounds, rheumatism, } \\
\text { inflammation }\end{array}$ & Marine/brackish/reef \\
\hline $\begin{array}{l}\text { Sphyrna mokarran (Rüppell, 1837), } \\
\text { great hammerhead }\end{array}$ & Osteoporosis & Marine/brackish/reef \\
\hline $\begin{array}{l}\text { Sphyrna zygaena (Linnaeus, 1758), } \\
\text { smooth hammerhead }\end{array}$ & Osteoporosis & Marine/brackish \\
\hline \multicolumn{3}{|l|}{ Squalidae } \\
\hline $\begin{array}{l}\text { Squalus cubensis Howell Rivero, } \\
\text { 1936, Cuban dogfish }\end{array}$ & Osteoporosis & Marine \\
\hline \multicolumn{3}{|l|}{ Rhinobatidae } \\
\hline $\begin{array}{l}\text { Rhinobatos percellens (Walbaum, } \\
\text { 1792), Chola guitarfish }\end{array}$ & Osteoporosis & Marine \\
\hline \multicolumn{3}{|l|}{ Centropomidae } \\
\hline $\begin{array}{l}\text { Centropomus parallelus Poey, 1860, } \\
\text { fat snook }\end{array}$ & Nephritis & Marine/brackish/freshwater \\
\hline $\begin{array}{l}\text { Centropomus undecimalis (Bloch, } \\
\text { 1792), common snook, "rubalão" }\end{array}$ & Edema in the legs & Marine/brackish/reef/freshwater \\
\hline \multicolumn{3}{|l|}{ Characidae } \\
\hline $\begin{array}{l}\text { Astyanax bimaculatus (Linnaeus, } \\
\text { 1758), twospot astyanax, "piaba-mirim," } \\
\text { "machadinha," "piaba chata" }\end{array}$ & $\begin{array}{l}\text { Alcoholism, leishmaniosis, skin } \\
\text { burns, wounds, rheumatism }\end{array}$ & Freshwater \\
\hline \multicolumn{3}{|l|}{ Clupeidae } \\
\hline $\begin{array}{l}\text { Harengula jaguana (Poey, 1865), } \\
\text { scaled herring, "sardinha" }\end{array}$ & Osteoporosis & Marine/brackish/reef \\
\hline $\begin{array}{l}\text { Opisthonema oglinum (Lesueur, } \\
\text { 1818), Atlantic thread herring, "sardinha" }\end{array}$ & Alcoholism, osteoporosis & Marine/reef \\
\hline \multicolumn{3}{|l|}{ Cynodontidae } \\
\hline $\begin{array}{l}\text { Hydrolycus scomberoides (Cuvier, } \\
\text { 1816), Payara, "cachorra" }\end{array}$ & Earache & Freshwater \\
\hline
\end{tabular}


TABle 1: Continued.

\begin{tabular}{|c|c|c|}
\hline Taxa & Health conditions treated & Habitat \\
\hline \multicolumn{3}{|l|}{ Dasyatidae } \\
\hline $\begin{array}{l}\text { Dasyatis guttata (Bloch \& Schneider, } \\
\text { 1801), longnose stingray, "raia branca" }\end{array}$ & $\begin{array}{c}\text { Asthma, pain relief in injuries } \\
\text { caused by the species' sting, } \\
\text { burns }\end{array}$ & Marine \\
\hline $\begin{array}{l}\text { Dasyatis marianae Gomes, Rosa \& } \\
\text { Gadig, 2000, Brazilian large-eyed } \\
\text { stingray, "raia mariquita," "raia de fogo" }\end{array}$ & $\begin{array}{l}\text { Asthma, pain relief in injuries } \\
\text { caused by the species' sting, } \\
\text { burns }\end{array}$ & Marine/reef \\
\hline \multicolumn{3}{|l|}{ Diodontidae } \\
\hline $\begin{array}{l}\text { Chilomycterus antillarum Jordan \& } \\
\text { Rutter, 1897, web burrfish }\end{array}$ & Wounds, lump & Marine/reef \\
\hline $\begin{array}{l}\text { Chilomycterus spinosus spinosus } \\
\text { (Linnaeus, 1758) }\end{array}$ & Wounds, lump & Marine/brackish \\
\hline \multicolumn{3}{|l|}{ Doradidae } \\
\hline $\begin{array}{l}\text { Franciscodoras marmoratus (Lütken, } \\
\text { 1874), "Urutu" }\end{array}$ & Injuries caused by itself & Freshwater \\
\hline $\begin{array}{l}\text { Lithodoras dorsalis (Valenciennes, } \\
\text { 1840), Rock-bacu }\end{array}$ & Swelling & Freshwater \\
\hline $\begin{array}{l}\text { Megalodoras uranoscopus } \\
\text { (Eigenmann \& Eigenmann, 1888), } \\
\text { "cuiu-cuiu” }\end{array}$ & Rheumatism & Freshwater \\
\hline $\begin{array}{l}\text { Oxydoras niger (Valenciennes, 1821), } \\
\text { ripsaw catfish, "cuiu-cuiu”" }\end{array}$ & Rheumatism & Freshwater \\
\hline $\begin{array}{l}\text { Platydoras costatus (Linnaeus, 1758), } \\
\text { Raphael catfish, "cuiu-cuiu”" }\end{array}$ & Rheumatism & Freshwater \\
\hline $\begin{array}{l}\text { Pterodoras granulosus (Valenciennes, } \\
\text { 1821), granulated catfish, "cuiu-cuiu" }\end{array}$ & Rheumatism & Freshwater \\
\hline \multicolumn{3}{|l|}{ Echeneidae } \\
\hline $\begin{array}{l}\text { Echeneis naucrates Linnaeus, 1758, } \\
\text { live sharksucker, "rêmora," "pegador" }\end{array}$ & Asthma, bronchitis & Marine/brackish/reef \\
\hline $\begin{array}{l}\text { Remora remora (Linnaeus, 1758), } \\
\text { shark sucker, "rêmora" }\end{array}$ & Osteoporosis & Marine/reef \\
\hline \multicolumn{3}{|l|}{ Erythrinidae } \\
\hline $\begin{array}{l}\text { Erythrinus erythrinus (Bloch \& } \\
\text { Schneider, 1801), "Matrôe" }\end{array}$ & Asthma & Freshwater \\
\hline $\begin{array}{l}\text { Hoplias malabaricus (Bloch, } \\
\text { 1794),trahira, "traíra" }\end{array}$ & $\begin{array}{l}\text { Ophthalmological problems, } \\
\text { rheumatism, cataracts, wounds, } \\
\text { snake bite, conjunctivitis, stroke, } \\
\text { thrombosis, asthma, toothache, } \\
\text { fever, earache, diarrhoea, } \\
\text { deafness, boils, bleeding, } \\
\text { alcoholism, tetanus, sore throat, } \\
\text { itching, sprains, leucoma }\end{array}$ & Freshwater \\
\hline Hoplias aimara (Valenciennes, 1847) & Earache & Freshwater \\
\hline \multicolumn{3}{|l|}{ Cichlidae } \\
\hline $\begin{array}{l}\text { Cichla melaniae Kullander \& } \\
\text { Ferreira, } 2006\end{array}$ & $\begin{array}{l}\text { Pain relief in injuries caused by } \\
\text { the ray sting }\end{array}$ & Freshwater \\
\hline \multicolumn{3}{|l|}{ Gadidae } \\
\hline $\begin{array}{l}\text { Gadus morhua Linnaeus, 1758, } \\
\text { Atlantic cod, "bacalhau" }\end{array}$ & Boils & Marine/brackish \\
\hline \multicolumn{3}{|l|}{ Ginglymostomatidae } \\
\hline $\begin{array}{l}\text { Ginglymostoma cirratum } \\
\text { (Bonnaterre, 1788),nurse shark, } \\
\text { "cação-lixa" }\end{array}$ & Rheumatism, osteoporosis & Marine/brackish/reef \\
\hline
\end{tabular}


TABLE 1: Continued.

\begin{tabular}{|c|c|c|}
\hline Taxa & Health conditions treated & Habitat \\
\hline \multicolumn{3}{|l|}{ Gymnotidae } \\
\hline $\begin{array}{l}\text { Electrophorus electricus (Linnaeus, } \\
\text { 1766), electric eel, “poraquê" }\end{array}$ & $\begin{array}{l}\text { Sprains, bruises, insect bites, } \\
\text { snake bite, asthma, flu, pain in } \\
\text { general, muscle strain, } \\
\text { rheumatism, osteoporosis, } \\
\text { deafness, pneumonia, itching }\end{array}$ & Freshwater \\
\hline \multicolumn{3}{|l|}{ Heptapteridae } \\
\hline $\begin{array}{c}\text { Pimelodella brasiliensis } \\
\text { (Steindachner, 1876), "mandim" }\end{array}$ & $\begin{array}{l}\text { Injuries caused by that fish } \\
\text { species }\end{array}$ & Freshwater \\
\hline \multicolumn{3}{|l|}{ Holocentridae } \\
\hline $\begin{array}{l}\text { Holocentrus adscensionis (Osbeck, } \\
\text { 1765), squirrelfish, "jaguaricá" }\end{array}$ & Wounds & Marine/reef \\
\hline \multicolumn{3}{|l|}{ Megalopidae } \\
\hline $\begin{array}{l}\text { Megalops atlanticus Valenciennes, } \\
\text { 1847, tarpon, "camurupim," } \\
\text { "cangurupim" }\end{array}$ & $\begin{array}{l}\text { Stroke, headache, asthma, } \\
\text { shortness of breath, thrombosis, } \\
\text { chest pain, injuries caused by } \\
\text { bang }\end{array}$ & Marine/brackish/reef/freshwater \\
\hline \multicolumn{3}{|l|}{ Monacanthidae } \\
\hline $\begin{array}{l}\text { Cantherhines macrocerus (Hollard, } \\
\text { 1853), American whitespotted filefish }\end{array}$ & Asthma & Marine/reef \\
\hline $\begin{array}{l}\text { Monacanthus ciliatus (Mitchill, } \\
\text { 1818), fringed filefish }\end{array}$ & Asthma & Marine/reef \\
\hline \multicolumn{3}{|l|}{ Muraenidae } \\
\hline $\begin{array}{l}\text { Gymnothorax funebris Ranzani, } \\
\text { 1839, green moray, "moréia verde" }\end{array}$ & Bleeding (wounds) & Marine/reef \\
\hline $\begin{array}{l}\text { Gymnothorax moringa (Cuvier, } \\
1829) \text {, spotted moray, "moréia pintada" }\end{array}$ & Bleeding (wounds) & Marine/reef \\
\hline $\begin{array}{l}\text { Gymnothorax vicinus (Castelnau, } \\
\text { 1855), purplemouth moray, "moréia" }\end{array}$ & Bleeding (wounds) & Marine/reef \\
\hline \multicolumn{3}{|l|}{ Myliobatidae } \\
\hline $\begin{array}{l}\text { Aetobatus narinari (Euphrasen, } \\
\text { 1790), spotted eagle ray, "raia-chita" }\end{array}$ & $\begin{array}{l}\text { Asthma, pain relief in injuries } \\
\text { caused by the species' sting, } \\
\text { burns, haemorrhage }\end{array}$ & Marine/reef \\
\hline \multicolumn{3}{|l|}{ Narcinidae } \\
\hline $\begin{array}{l}\text { Narcine bancroftii (Griffith \& Smith, } \\
\text { 1834), lesser electric ray }\end{array}$ & Pain & Marine \\
\hline $\begin{array}{l}\text { Narcine brasiliensis (Olfers, 1831), } \\
\text { Brazilian electric ray, "raia elétrica" }\end{array}$ & Toothache & Marine/reef \\
\hline \multicolumn{3}{|l|}{ Pimelodidae } \\
\hline $\begin{array}{l}\text { Phractocephalus hemioliopterus } \\
\text { (Bloch \& Schneider, 1801), redtail catfish, } \\
\text { "Pirarara" }\end{array}$ & $\begin{array}{l}\text { Asthma, wounds, hernia, burns } \\
\text { in the skin, rheumatism, flu, } \\
\text { cough }\end{array}$ & Freshwater \\
\hline $\begin{array}{l}\text { Pseudoplatystoma corruscans (Spix \& } \\
\text { Agassiz, 1829), spotted sorubim, } \\
\text { "surubim" }\end{array}$ & Flu, removal of wrath & Freshwater \\
\hline $\begin{array}{l}\text { Pseudoplatystoma fasciatum } \\
\text { (Lunnaeus, 1776), barred sorubim, } \\
\text { "surubim" }\end{array}$ & Cold & Freshwater \\
\hline $\begin{array}{l}\text { Sorubimichthys planiceps (Spix \& } \\
\text { Agassiz, 1829), firewood catfish, "surubim } \\
\text { chicote" }\end{array}$ & Leishmaniosis, tuberculosis & Freshwater \\
\hline $\begin{array}{l}\text { Zungaro zungaro (Humboldt, 1821), } \\
\text { gilded catfish, black manguruyu, “jaú” }\end{array}$ & $\begin{array}{l}\text { Asthma, toothache, earache, } \\
\text { wounds, athlete's foot, burns in } \\
\text { the skin, rheumatism, flu }\end{array}$ & Freshwater \\
\hline
\end{tabular}


TABle 1: Continued.

\begin{tabular}{|c|c|c|}
\hline Taxa & Health conditions treated & Habitat \\
\hline \multicolumn{3}{|l|}{ Potamotrygonidae } \\
\hline $\begin{array}{l}\text { Paratrygon aiereba (Müller \& Henle, } \\
\text { 1841), discus ray,"arraia” }\end{array}$ & $\begin{array}{l}\text { Asthma, hernia, flu, pneumonia, } \\
\text { cough, earache, burns }\end{array}$ & Freshwater \\
\hline $\begin{array}{l}\text { Plesiotrygon iwamae Rosa, Castello } \\
\text { \& Thorson, 1987, long-tailed river stingray }\end{array}$ & $\begin{array}{l}\text { Pain relief in injuries caused by } \\
\text { the species' sting, wounds, cracks } \\
\text { in the sole of the feet }\end{array}$ & Freshwater \\
\hline $\begin{array}{l}\text { Potamotrygon hystrix (Müller \& } \\
\text { Henle, 1834),porcupine river stingray, } \\
\text { "arraia" }\end{array}$ & $\begin{array}{l}\text { Asthma, hernia, flu, pneumonia, } \\
\text { cough, earache, burns }\end{array}$ & Freshwater \\
\hline $\begin{array}{l}\text { Potamotrygon motoro (Müller \& } \\
\text { Henle, 1841), South American freshwater } \\
\text { stingray, ocellate river stingray, "arraia" }\end{array}$ & $\begin{array}{l}\text { Asthma, hernia, flu, pneumonia, } \\
\text { cough, earache, burns }\end{array}$ & Freshwater \\
\hline $\begin{array}{l}\text { Potamotrygon orbignyi (Castelnau, } \\
\text { 1855), smooth back river stingray, "arraia" }\end{array}$ & $\begin{array}{l}\text { Pain relief in injuries caused by } \\
\text { that species' sting }\end{array}$ & Freshwater \\
\hline \multicolumn{3}{|l|}{ Pristidae } \\
\hline $\begin{array}{l}\text { Pristis pectinata Latham, } 1794, \\
\text { smalltooth sawfish, "espadarte," } \\
\text { "peixe-serra" }\end{array}$ & Asthma, rheumatism, arthritis & Marine/brackish/freshwater \\
\hline $\begin{array}{l}\text { Pristis perotteti Müller \& Henle, } \\
\text { 1841, largetooth sawfish, "espadarte" }\end{array}$ & Asthma, rheumatism, arthritis & Marine/brackish/freshwater \\
\hline \multicolumn{3}{|l|}{ Prochilodontidae } \\
\hline $\begin{array}{l}\text { Prochilodus argenteus Spix \& } \\
\text { Agassiz, 1829, "curimatá-pacú," } \\
\text { "curimatá" }\end{array}$ & $\begin{array}{l}\text { To avoid swelling of the breast } \\
\text { feeding, mycosis }\end{array}$ & Freshwater \\
\hline $\begin{array}{l}\text { Prochilodus nigricans Spix \& Agassiz, } \\
\text { 1829, black prochilodus, "curimatã" }\end{array}$ & $\begin{array}{l}\text { Chilblain, skin burns, wounds, } \\
\text { rheumatism, eye pains }\end{array}$ & Freshwater \\
\hline \multicolumn{3}{|l|}{ Serrasalmidae } \\
\hline $\begin{array}{l}\text { Colossoma macropomum (Cuvier, } \\
\text { 1818), cachama, "tambaqui" }\end{array}$ & Paralysis of arms and legs & Freshwater \\
\hline $\begin{array}{l}\text { Mylossoma duriventre (Cuvier, 1818), } \\
\text { pacupeba, "pacu-manteiga" }\end{array}$ & Venereal disease & Freshwater \\
\hline $\begin{array}{l}\text { Serrasalmus brandtii Lütken, } 1875 \text {, } \\
\text { white piranha, "pirambeba" }\end{array}$ & Inflammations, sexual impotence & Freshwater \\
\hline \multicolumn{3}{|l|}{ Sciaenidae } \\
\hline $\begin{array}{l}\text { Cynoscion acoupa (Lacepède, 1801), } \\
\text { acoupa weakfish, "pescada amarela" }\end{array}$ & Renal failure & Marine/brackish/freshwater \\
\hline $\begin{array}{l}\text { Cynoscion leiarchus (Cuvier, 1830), } \\
\text { smooth weakfish, "pescada branca" }\end{array}$ & Renal failure & Marine/brackish \\
\hline $\begin{array}{l}\text { Micropogonias furnieri (Desmarest, } \\
\text { 1823), whitemouth croaker, "corvina" }\end{array}$ & $\begin{array}{l}\text { Pain relief in injuries caused by } \\
\text { the species' sting, cough, asthma, } \\
\text { bronchitis }\end{array}$ & Marine/brackish \\
\hline $\begin{array}{l}\text { Pachyurus francisci (Cuvier, 1830), } \\
\text { San Francisco croaker, "cruvina-de-bico" }\end{array}$ & $\begin{array}{l}\text { Asthma, urinary incontinence, } \\
\text { backache }\end{array}$ & Freshwater \\
\hline $\begin{array}{l}\text { Plagioscion squamosissimus (Heckel, } \\
\text { 1840), South American silver croaker, } \\
\text { "curvina" }\end{array}$ & $\begin{array}{l}\text { Urinary disorders, haemorrhage, } \\
\text { snake bites }\end{array}$ & Freshwater \\
\hline $\begin{array}{l}\text { Plagioscion surinamensis (Bleeker, } \\
\text { 1873), Bashaw,"pacora,” “curvina”" }\end{array}$ & $\begin{array}{l}\text { Urinary disorders, haemorrhage, } \\
\text { snake bites }\end{array}$ & Freshwater \\
\hline \multicolumn{3}{|l|}{ Sparidae } \\
\hline $\begin{array}{l}\text { Calamus penna (Valenciennes, } \\
\text { 1830), sheepshead porgy, "peixe-pena" }\end{array}$ & Asthma & Marine/reef \\
\hline
\end{tabular}


TABle 1: Continued.

\begin{tabular}{|c|c|c|}
\hline Taxa & Health conditions treated & Habitat \\
\hline \multicolumn{3}{|l|}{ Synbranchidae } \\
\hline $\begin{array}{l}\text { Synbranchus marmoratus Bloch, } \\
\text { 1795, marbled swamp eel,"muçum” }\end{array}$ & Bronchitis & Freshwater/brackish \\
\hline \multicolumn{3}{|l|}{ Tetraodontidae } \\
\hline $\begin{array}{l}\text { Colomesus psittacus (Bloch \& } \\
\text { Schneider, 1801), banded puffer, "baiacu" }\end{array}$ & Breast cancer, backache, warts & Marine/brackish/freshwater \\
\hline $\begin{array}{l}\text { Sphoeroides testudineus (Linnaeus, } \\
\text { 1758), checkered puffer, "baiacu" }\end{array}$ & Rheumatism & Marine/brackish/reef \\
\hline \multicolumn{3}{|l|}{ Trichiuridae } \\
\hline $\begin{array}{l}\text { Trichiurus lepturus Linnaeus, } 1758 \text {, } \\
\text { largehead hairtail }\end{array}$ & Asthma & Marine/brackish \\
\hline \multicolumn{3}{|l|}{ Urotrygonidae } \\
\hline $\begin{array}{l}\text { Urotrygon microphthalmum } \\
\text { Delsman, 1941, smalleyed round stingray, } \\
\text { "raia" }\end{array}$ & $\begin{array}{l}\text { Asthma, pain relief in injuries caused } \\
\text { by the species' sting, burns }\end{array}$ & Marine \\
\hline \multicolumn{3}{|l|}{ Amphibians } \\
\hline \multicolumn{3}{|l|}{ Bufonidae } \\
\hline Rhinella jimi (Stevaux 2002) & $\begin{array}{l}\text { Urinary incontinence, dental caries, } \\
\text { cancer, wounds, boils, erysipelas acne, } \\
\text { inducing abortion }\end{array}$ & Semiaquatic \\
\hline \multicolumn{3}{|l|}{ Leptodactylidae } \\
\hline $\begin{array}{l}\text { Leptodactylus cf. labyrinthicus (Spix, } \\
\text { 1824), South American pepper frog, } \\
\text { "jia-de-peito," "rã- pimenta" }\end{array}$ & $\begin{array}{l}\text { Earache, rheumatism, joint pain, } \\
\text { cancer, sore throat }\end{array}$ & Semiaquatic \\
\hline $\begin{array}{l}\text { Leptodactylus vastus Lutz, } 1930 \text {, } \\
\text { South American pepper frog, ra-pimenta }\end{array}$ & $\begin{array}{l}\text { Sore throat, cough, asthma, arthritis, } \\
\text { backache, tonsillitis, hoarseness }\end{array}$ & Semiaquatic \\
\hline \multicolumn{3}{|l|}{ Reptiles } \\
\hline \multicolumn{3}{|l|}{ Iguanidae } \\
\hline $\begin{array}{l}\text { Iguana iguana (Linnaeus, 1758), } \\
\text { Common iguana, "camaleão" }\end{array}$ & $\begin{array}{l}\text { Earache, erysipelas, asthma, } \\
\text { rheumatism, edema, abscesses, joint } \\
\text { pain, wounds, acne, athlete's foot, sore } \\
\text { throat, swelling, burn, tumour, sucking } \\
\text { a splinter out of skin or flesh, boil, } \\
\text { injuries caused by the spines of the } \\
\text { "arraia" and others fishes, } \\
\text { inflammation, hernia }\end{array}$ & Terrestrial \\
\hline \multicolumn{3}{|l|}{ Teiidae } \\
\hline $\begin{array}{l}\text { Tupinambis merianae (Duméril \& } \\
\text { Bibron, 1839), Lizard, "tegu," “tejuaçú" }\end{array}$ & $\begin{array}{l}\text { Earache, deafness, rheumatism, } \\
\text { erysipelas, skin thorns and wounds, } \\
\text { respiratory diseases, sore throat, snake } \\
\text { bite, asthma, tumour, swelling, } \\
\text { infection, bronchitis }\end{array}$ & Terrestrial \\
\hline $\begin{array}{l}\text { Tupinambis teguixin (Linnaeus } \\
\text { 1758), Lizard, "tegu," "tejuaçú" }\end{array}$ & $\begin{array}{l}\text { Sexual impotence, rheumatism, } \\
\text { erysipelas, dermatitis, snake bites, } \\
\text { asthma, tetanus, earache, thrombosis, } \\
\text { wounds, infection of nail, swelling, } \\
\text { herpes zoster, irritation when milk } \\
\text { teeth are erupting, jaundice, } \\
\text { inflammation, tumour, sore throat, } \\
\text { infection, bronchitis, injuries caused } \\
\text { by the spines of the "arraia," pain relief } \\
\text { in injuries caused by snake bites, } \\
\text { toothache, sucking a splinter out of } \\
\text { skin or flesh, headache, cough, stroke, } \\
\text { coarse throat }\end{array}$ & Terrestrial \\
\hline
\end{tabular}


TABle 1: Continued.

\begin{tabular}{|c|c|c|}
\hline Taxa & Health conditions treated & Habitat \\
\hline \multicolumn{3}{|l|}{ Boidae } \\
\hline $\begin{array}{l}\text { Boa constrictor (Linnaeus, 1758), } \\
\text { Boa, "jibóia” }\end{array}$ & $\begin{array}{l}\text { Rheumatism, lung disease, } \\
\text { thrombosis, boils, tuberculosis, } \\
\text { stomach ache, edema, snake bite, } \\
\text { cancer, ache, swelling, helping to } \\
\text { prevent abortion, pain in the body, } \\
\text { inflammation, athlete's foot, } \\
\text { calluses, tumours, cracks in the } \\
\text { sole of the feet, goitre, sore throat, } \\
\text { arthrosis, insect sting, dog bite, } \\
\text { erysipelas, thrombosis, asthma, } \\
\text { neck strain, strain muscle }\end{array}$ & Terrestrial \\
\hline $\begin{array}{l}\text { Corallus hortolanus (Linnaeus, 1758), } \\
\text { snake }\end{array}$ & $\begin{array}{l}\text { Assisting in removing spines or } \\
\text { other sharp structures from the } \\
\text { skin, rheumatism }\end{array}$ & Terrestrial \\
\hline $\begin{array}{l}\text { Eunectes murinus (Linnaeus, 1758), } \\
\text { anaconda, "sucurujú," “sucuri”" }\end{array}$ & $\begin{array}{l}\text { Wounds, skin problems, bruises, } \\
\text { sprains, arthrosis, rheumatism, } \\
\text { boils, sexual impotence, headache, } \\
\text { sore throat, thrombosis, swelling, } \\
\text { tumour, asthma, muscle strain, } \\
\text { numbness, syphilis, reducing pain, } \\
\text { luxation }\end{array}$ & Semiaquatic \\
\hline $\begin{array}{l}\text { Epicrates cenchria (Linnaeus, 1758), } \\
\text { Brazilian rainbow boa, "salamanta" }\end{array}$ & $\begin{array}{l}\text { Rheumatism, pain in articulations, } \\
\text { injuries caused by itself, sore } \\
\text { throat, earache }\end{array}$ & Terrestrial \\
\hline \multicolumn{3}{|l|}{ Crotalidae } \\
\hline $\begin{array}{l}\text { Crotalus durissus (Linnaeus, 1758), } \\
\text { Neotropical rattlesnake, "cascavel" }\end{array}$ & $\begin{array}{l}\text { Asthma, snake bite, thrombosis, } \\
\text { wounds, luxation, rheumatism, } \\
\text { pain in the legs, erysipelas, } \\
\text { deafness, epilepsy, skin diseases, } \\
\text { tuberculosis, hanseniasis, } \\
\text { backache, tumour, boil, headache, } \\
\text { earache, osteoporosis, sore throat, } \\
\text { toothache, pain relief in injuries } \\
\text { caused by sting of insects and } \\
\text { snake bite, irritation when milk } \\
\text { teeth are erupting, tonsillitis, } \\
\text { impotence, fatigue }\end{array}$ & \\
\hline \multicolumn{3}{|l|}{ Chelidae } \\
\hline $\begin{array}{l}\text { Phrynops geoffroanus (Schweigger, } \\
\text { 1812), Geoffroy's side-necked turtle, } \\
\text { "cágado" }\end{array}$ & $\begin{array}{l}\text { Asthma, sore throat, swelling, } \\
\text { earache, rheumatism, arthrosis, } \\
\text { healing of umbilical cord of } \\
\text { newborn baby, mumps }\end{array}$ & Freshwater \\
\hline Phrynops tuberosus (Peters, 1870) & $\begin{array}{l}\text { Diphtheria, headache, earache, } \\
\text { pain in the breast, wounds, } \\
\text { furuncle, gastritis, swelling, } \\
\text { haemorrhoids, sore throat, } \\
\text { backache, eye problems, sucking a } \\
\text { splinter out of skin or flesh, } \\
\text { rheumatism, deafness }\end{array}$ & Freshwater \\
\hline $\begin{array}{l}\text { Mesoclemmys tuberculata } \\
\text { (Luederwaldt, 1926), tuberculate } \\
\text { toadhead turtle, "cágado," } \\
\text { "cágado-d'água" }\end{array}$ & $\begin{array}{c}\text { Rheumatism, discharge, } \\
\text { thrombosis, bronchitis, diarrhoea, } \\
\text { haemorrhage, asthma, sore throat, } \\
\text { hoarseness, muscle aches }\end{array}$ & Freshwater \\
\hline
\end{tabular}


TABle 1: Continued.

\begin{tabular}{|c|c|c|}
\hline Taxa & Health conditions treated & Habitat \\
\hline \multicolumn{3}{|l|}{ Cheloniidae } \\
\hline $\begin{array}{l}\text { Caretta caretta (Linnaeus, 1758), } \\
\text { loggerhead turtle, "tartaruga cabeçuda" }\end{array}$ & $\begin{array}{c}\text { Injuries caused by bang, } \\
\text { toothache, diabetes, headache, } \\
\text { backache, wounds, cough, } \\
\text { bronchitis, asthma, thrombosis, } \\
\text { rheumatism, stroke, hoarseness, } \\
\text { flu, backache, earache, sore throat, } \\
\text { swelling }\end{array}$ & Marine \\
\hline $\begin{array}{l}\text { Chelonia mydas (Linnaeus, 1758), } \\
\text { green sea turtle, "tartaruga verde," } \\
\text { "aruanã" }\end{array}$ & $\begin{array}{l}\text { Injuries caused by bang, } \\
\text { toothache, diabetes, headache, } \\
\text { backache, wounds, cough, } \\
\text { bronchitis, asthma, flu, } \\
\text { thrombosis, rheumatism, } \\
\text { toothache, stroke, hoarseness, } \\
\text { earache, sore throat, swelling, } \\
\text { whooping cough, arthritis, } \\
\text { erysipelas, boil, wounds, arthrosis, } \\
\text { inflammation }\end{array}$ & Marine \\
\hline $\begin{array}{l}\text { Eretmochelys imbricata (Linnaeus, } \\
\text { 1766), Atlantic hawksbill, "tartaruga de } \\
\text { pente" }\end{array}$ & $\begin{array}{c}\text { Injuries caused by bang, } \\
\text { toothache, diabetes, headache, } \\
\text { backache, wounds, cough, } \\
\text { bronchitis, asthma, thrombosis, } \\
\text { stroke, hoarseness, flu, } \\
\text { rheumatism, earache, sore throat, } \\
\text { swelling }\end{array}$ & Marine \\
\hline $\begin{array}{l}\text { Lepidochelys olivacea (Eschscholtz, } \\
\text { 1829), olive ridley }\end{array}$ & $\begin{array}{l}\text { Injuries caused by bang, } \\
\text { toothache, diabetes, headache, } \\
\text { backache, wounds, cough, flu, } \\
\text { bronchitis, asthma, thrombosis, } \\
\text { rheumatism, stroke, hoarseness }\end{array}$ & Marine \\
\hline \multicolumn{3}{|l|}{ Dermochelyidae } \\
\hline $\begin{array}{l}\text { Dermochelys coriacea (Vandelli, } \\
\text { 1761), leatherback turtle, "tartaruga de } \\
\text { couro" }\end{array}$ & $\begin{array}{l}\text { Rheumatism, earache, sore throat, } \\
\text { swelling }\end{array}$ & Marine \\
\hline \multicolumn{3}{|l|}{ Geoemydidae } \\
\hline $\begin{array}{l}\text { Rhinoclemmys punctularia (Daudin, } \\
\text { 1802), spot-legged turtle }\end{array}$ & $\begin{array}{l}\text { Wounds, tumour, erysipelas, } \\
\text { earache, rheumatism }\end{array}$ & Semiaquatic \\
\hline \multicolumn{3}{|l|}{ Podocnemididae } \\
\hline $\begin{array}{l}\text { Podocnemis expansa (Schweiger, } \\
\text { 1812), Amazon river turtle, "tartaruga da } \\
\text { amazônia" }\end{array}$ & $\begin{array}{l}\text { Inflammation, acne, tumour, boil, } \\
\text { rheumatism, pterygium, skin } \\
\text { spots, backache, earache, arthrosis, } \\
\text { arthritis, swelling, wrinkle }\end{array}$ & Freshwater \\
\hline $\begin{array}{l}\text { Podocnemis unifilis (Troschel, 1848), } \\
\text { yellow-spotted river turtle, "tracajá" }\end{array}$ & $\begin{array}{l}\text { Wounds, tumour, erysipelas, } \\
\text { earache, rheumatism }\end{array}$ & Freshwater \\
\hline $\begin{array}{l}\text { Podocnemis sextuberculata Cornalia, } \\
\text { 1849, six-tubercled Amazon River turtle }\end{array}$ & Blackhead, acne & Freshwater \\
\hline $\begin{array}{l}\text { Peltocephalus dumeriliana } \\
\text { Schweigger 1812, "Cabeçuda" }\end{array}$ & Blackhead, acne & Freshwater \\
\hline \multicolumn{3}{|l|}{ Testudinidae } \\
\hline $\begin{array}{l}\text { Chelonoidis carbonaria (Spix, 1824), } \\
\text { red-footed tortoise, “jabuti” }\end{array}$ & $\begin{array}{l}\text { Catarrh, erysipelas, bronchitis, } \\
\text { stopping the sensation of getting } \\
\text { thirsty, asthma }\end{array}$ & Terrestrial \\
\hline $\begin{array}{l}\text { Chelonoidis denticulata (Linnaeus, } \\
\text { 1766), yellow-footed tortoise, "jabuti" }\end{array}$ & $\begin{array}{c}\text { Sore throat, rheumatism, hernia, } \\
\text { wounds, leishmaniosis, varicocele, } \\
\text { earache }\end{array}$ & Terrestrial \\
\hline
\end{tabular}


TABle 1: Continued.

\begin{tabular}{|c|c|c|}
\hline Taxa & Health conditions treated & Habitat \\
\hline \multicolumn{3}{|l|}{ Kinosternidae } \\
\hline $\begin{array}{l}\text { Kinosternon acutum (Linnaeus } \\
\text { 1766), Tabasco Mud Turtle }\end{array}$ & Muscle aches & Freshwater \\
\hline \multicolumn{3}{|l|}{ Alligatoridae } \\
\hline $\begin{array}{l}\text { Caiman crocodilus (Linnaeus, 1758), } \\
\text { common cayman, "jacaré tinga" }\end{array}$ & $\begin{array}{l}\text { Asthma, stroke, bronchitis, } \\
\text { backache, earache, rheumatism, } \\
\text { thrombosis, sexual impotence, } \\
\text { snake bites (antidote), evil eye, } \\
\text { irritation when milk teeth are } \\
\text { erupting, discharge, swelling, } \\
\text { scratch, athlete's foot, } \\
\text { ophthalmological problems, } \\
\text { asthma, sore throat, amulet used } \\
\text { as a protection against snake bite, } \\
\text { rheumatism, hernia, prostate } \\
\text { problems, infection, thrombosis }\end{array}$ & Freshwater \\
\hline $\begin{array}{l}\text { Caiman latirostris (Daudin, 1801), } \\
\text { broad-snouted caiman, } \\
\text { "jacaré-do-papo-amarelo" }\end{array}$ & $\begin{array}{l}\text { Asthma, sore throat, amulet used } \\
\text { as a protection against snake bite, } \\
\text { rheumatism, irritation when } \\
\text { milk teeth are erupting, hernia, } \\
\text { prostate problems }\end{array}$ & Freshwater \\
\hline $\begin{array}{l}\text { Melanosuchus niger (Spix, 1825), } \\
\text { black caiman, "jacare açú" }\end{array}$ & $\begin{array}{l}\text { Thrombosis, infection, swelling, } \\
\text { asthma, amulet used as a } \\
\text { protection against snake bite, } \\
\text { injuries caused by spines of the } \\
\text { "arraia," pain relief in injuries } \\
\text { caused by snake bites }\end{array}$ & Freshwater \\
\hline $\begin{array}{l}\text { Paleosuchus palpebrosus (Cuvier, } \\
\text { 1807), dwarf caiman, “jacaré coroa," } \\
\text { "jacaré," “jacaré-preto," “crocodilo" }\end{array}$ & $\begin{array}{l}\text { Snake bite, asthma, stroke, } \\
\text { rheumatism, thrombosis, } \\
\text { backache, sexual impotence, } \\
\text { edema, mycosis, evil eye, } \\
\text { irritation when milk teeth are } \\
\text { erupting, snake bite (antidote), } \\
\text { discharge, sore throat, amulet } \\
\text { used as a protection against snake } \\
\text { bite, hernia, prostate problems }\end{array}$ & Freshwater \\
\hline $\begin{array}{l}\text { Paleosuchus trigonatus (Schneider, } \\
\text { 1801), smooth-fronted caiman, "Jacaré } \\
\text { coroa" }\end{array}$ & Rheumatism & Freshwater \\
\hline \multicolumn{3}{|l|}{ Birds } \\
\hline \multicolumn{3}{|l|}{ Anatidae } \\
\hline $\begin{array}{l}\text { Sarkidiornis sylvicola H. Ihering \& R. } \\
\text { Ihering, 1907, American Comb Duck, } \\
\text { "putrião" }\end{array}$ & Bleeding (wounds) & Terrestrial \\
\hline \multicolumn{3}{|l|}{ Anhimidae } \\
\hline $\begin{array}{l}\text { Anhima cornuta (Linnaeus, 1766), } \\
\text { horned screamer, "anuhma" }\end{array}$ & $\begin{array}{l}\text { Intoxication from poisonous } \\
\text { animals }\end{array}$ & Terrestrial \\
\hline \multicolumn{3}{|l|}{ Ardeidae } \\
\hline $\begin{array}{l}\text { Ardea cocoi (Linnaeus, 1766), } \\
\text { white-necked Heron }\end{array}$ & $\begin{array}{l}\text { Swelling, inflammation, injuries } \\
\text { caused by the spines of the } \\
\text { "arraia" and others fishes, } \\
\text { asthma, boil, tumour, } \\
\text { inflammation, rheumatism, } \\
\text { earache }\end{array}$ & Terrestrial \\
\hline \multicolumn{3}{|l|}{ Caprimulgidae } \\
\hline $\begin{array}{l}\text { Nyctidromus albicollis (Gmelin, } \\
\text { 1789), pauraque, "bacurau" }\end{array}$ & Amulets, snake bite & Terrestrial \\
\hline
\end{tabular}


TABle 1: Continued.

\begin{tabular}{|c|c|c|}
\hline Taxa & Health conditions treated & Habitat \\
\hline \multicolumn{3}{|l|}{ Cracidae } \\
\hline $\begin{array}{l}\text { Penelope jacucaca (Spix, 1825), } \\
\text { white-browed guan, "jacu” }\end{array}$ & Insomnia, epilepsy & Terrestrial \\
\hline $\begin{array}{l}\text { Pauxi tuberosa (Spix, 1825), } \\
\text { razor-billed Curassow }\end{array}$ & $\begin{array}{l}\text { Bleeding, snakebite, indigestion, } \\
\text { stroke, lack of appetite in } \\
\text { children, pneumonia }\end{array}$ & Terrestrial \\
\hline \multicolumn{3}{|l|}{ Ciconiidae } \\
\hline $\begin{array}{l}\text { Ciconia maguari (Gmelin, 1789), } \\
\text { maguari stork }\end{array}$ & $\begin{array}{l}\text { Injuries caused by the spines of } \\
\text { the "arraia" and others fishes, } \\
\text { thrombosis }\end{array}$ & Terrestrial \\
\hline \multicolumn{3}{|l|}{ Columbidae } \\
\hline $\begin{array}{l}\text { Columbina minuta (Linnaeus, 1766), } \\
\text { plain-breasted ground dove }\end{array}$ & $\begin{array}{l}\text { Lack of appetite, nausea during } \\
\text { pregnancy }\end{array}$ & Terrestrial \\
\hline $\begin{array}{l}\text { Columbina picui (Temminck, 1813), } \\
\text { Picui Dove }\end{array}$ & $\begin{array}{l}\text { Lack of appetite, nausea during } \\
\text { pregnancy, deafness }\end{array}$ & Terrestrial \\
\hline $\begin{array}{l}\text { Columbina talpacoti (Temminck, } \\
\text { 1810), Ruddy Ground Dove, } \\
\text { "rolinha-caldo-de-feijão" }\end{array}$ & $\begin{array}{l}\text { Lack of appetite, nausea during } \\
\text { pregnancy, deafness }\end{array}$ & Terrestrial \\
\hline $\begin{array}{l}\quad \text { Leptotila rufaxilla (Richard \& } \\
\text { Bernard, 1792), Grey-Fronted Dove, } \\
\text { "juriti” }\end{array}$ & $\begin{array}{c}\text { Lack of appetite, nausea during } \\
\text { pregnancy, deafness, stye, } \\
\text { thrombosis }\end{array}$ & Terrestrial \\
\hline \multicolumn{3}{|l|}{ Corvidae } \\
\hline $\begin{array}{l}\text { Cyanocorax cyanopogon (Wied, } \\
\text { 1821), white-naped jay, "can-can" }\end{array}$ & Asthma & Terrestrial \\
\hline \multicolumn{3}{|l|}{ Cuculidae } \\
\hline $\begin{array}{l}\text { Crotophaga ani Linnaeus, } 1758 \text {, } \\
\text { smooth-billed ani }\end{array}$ & $\begin{array}{l}\text { Bronchitis, thrombosis, asthma, } \\
\text { whooping cough }\end{array}$ & Terrestrial \\
\hline $\begin{array}{l}\text { Guira guira (Gmelin, 1788), guira } \\
\text { cuckoo, "anum branco" }\end{array}$ & Asthma & Terrestrial \\
\hline \multicolumn{3}{|l|}{ Charadriidae } \\
\hline $\begin{array}{l}\text { Vanellus chilensis (Molina, 1782), } \\
\text { southern lapwing, "quero-quero" }\end{array}$ & Helping to stay awake & Terrestrial \\
\hline \multicolumn{3}{|l|}{ Emberizidae } \\
\hline $\begin{array}{l}\text { Coereba flaveola (Linnaeus, 1758), } \\
\text { bananaquit, "caga-sebo" }\end{array}$ & Thrombosis & Terrestrial \\
\hline \multicolumn{3}{|l|}{ Furnariidae } \\
\hline $\begin{array}{l}\text { Furnarius rufus (Gmelin, 1788), } \\
\text { rufous hornero, "maria-barreira" }\end{array}$ & Mumps & Terrestrial \\
\hline \multicolumn{3}{|l|}{ Podicipedidae } \\
\hline $\begin{array}{l}\text { Tachybaptus dominicus (Linnaeus, } \\
\text { 1766), Least Grebe, } \\
\text { "mergulhão-pequeno," “mergulhão," } \\
\text { "mergulhão-preto" }\end{array}$ & Eye problems & Semiaquatic \\
\hline \multicolumn{3}{|l|}{ Rallidae } \\
\hline $\begin{array}{l}\text { Aramides cajanea (Statius Müller, } \\
\text { 1776), grey-necked wood-rail, "saracura" }\end{array}$ & Evil eye & Terrestrial \\
\hline \multicolumn{3}{|l|}{ Rheidae } \\
\hline $\begin{array}{l}\text { Rhea americana (Linnaeus, 1758), } \\
\text { greater rhea, "ema" }\end{array}$ & $\begin{array}{l}\text { General aches, rheumatism, } \\
\text { thrombosis, strokes }\end{array}$ & Terrestrial \\
\hline
\end{tabular}


TABle 1: Continued.

\begin{tabular}{|c|c|c|}
\hline Taxa & Health conditions treated & Habitat \\
\hline \multicolumn{3}{|l|}{ Tinamidae } \\
\hline $\begin{array}{l}\text { Crypturellus noctivagus (Wied, } \\
\text { 1820), yellow-legged tinamou,"zabele" }\end{array}$ & Thrombosis, stroke & Terrestrial \\
\hline $\begin{array}{l}\text { Crypturellus tataupa (Temminck, } \\
\text { 1815), Tataupa Tinamou }\end{array}$ & $\begin{array}{l}\text { Assisting children who take longer } \\
\text { than usual to start walking }\end{array}$ & Terrestrial \\
\hline $\begin{array}{l}\text { Crypturellus parvirostris (Wagler, } \\
\text { 1827), small-billed Tinamou }\end{array}$ & $\begin{array}{l}\text { Assisting children who take longer } \\
\text { than usual to start walking }\end{array}$ & Terrestrial \\
\hline $\begin{array}{l}\text { Nothura boraquira (Spix, 1825), } \\
\text { white-bellied nothura, "codorna" }\end{array}$ & Thrombosis, stroke, earache & Terrestrial \\
\hline $\begin{array}{l}\text { Nothura maculosa (Temminck, } \\
\text { 1815), Spotted Nothura, "lambú } \\
\text { espanta-boiada," "lambú-de-capoeira" }\end{array}$ & Snake bite & Terrestrial \\
\hline $\begin{array}{l}\text { Tinamus tao Temminck, 1815, Grey } \\
\text { Tinamou }\end{array}$ & Snake bite & Terrestrial \\
\hline $\begin{array}{l}\text { Rhynchotus rufescens (Temminck, } \\
\text { 1815), red-winged tinamou,"perdiz" }\end{array}$ & Thrombosis, snake bites (antidote) & Terrestrial \\
\hline \multicolumn{3}{|l|}{ Mammals } \\
\hline \multicolumn{3}{|l|}{ Agoutidae } \\
\hline $\begin{array}{l}\text { Cuniculus paca (Linnaeus, 1766), } \\
\text { spotted paca, "paca” }\end{array}$ & $\begin{array}{l}\text { Wound in the breast caused by } \\
\text { suckling, ophthalmological problems, } \\
\text { stomach disorders, pterygium, sucking } \\
\text { a splinter out of skin or flesh, injuries } \\
\text { caused by the spines of "arraia," } \\
\text { control of cholesterol level }\end{array}$ & Terrestrial \\
\hline \multicolumn{3}{|l|}{ Bovidae } \\
\hline $\begin{array}{l}\text { Bubalus bubalis (Linnaeus, 1758), } \\
\text { water buffalo (feral), "búfalo" }\end{array}$ & Rheumatism, osteoporosis, thrombosis & Terrestrial \\
\hline \multicolumn{3}{|l|}{ Bradypodidae } \\
\hline $\begin{array}{l}\text { Bradypus variegatus Shinz, } 1825, \\
\text { brown-throated three-toed sloth, } \\
\text { "Preguiça pequena" }\end{array}$ & Thrombosis & Terrestrial \\
\hline $\begin{array}{l}\text { Bradypus tridactylus Linnaeus, } 1758, \\
\text { pale-throated three-toed sloth, "Preguiça" }\end{array}$ & Thrombosis, insects bite, scorpions bite & Terrestrial \\
\hline \multicolumn{3}{|l|}{ Canidae } \\
\hline $\begin{array}{l}\text { Cerdocyon thous (Linnaeus, 1766), } \\
\text { crab-eating fox, "raposa" }\end{array}$ & $\begin{array}{l}\text { Rheumatism, flu, haemorrhoids, } \\
\text { disorders after parturition (to } \\
\text { accelerate recovery after parturition) }\end{array}$ & Terrestrial \\
\hline $\begin{array}{l}\text { Chrysocyon brachyurus (Illiger, } \\
\text { 1815), maned wolf, "lobo-guará" }\end{array}$ & Epilepsy & Terrestrial \\
\hline $\begin{array}{l}\text { Dusicyon thous Linnaeus, 1766, } \\
\text { crab-eating fox, "raposa" }\end{array}$ & $\begin{array}{l}\text { Alcoholism, thrombosis, rheumatism, } \\
\text { ophthalmological problems, diabetes, } \\
\text { urinary infection }\end{array}$ & Terrestrial \\
\hline \multicolumn{3}{|l|}{ Caviidae } \\
\hline $\begin{array}{l}\text { Cavia aperea Erxleben, 1777, } \\
\text { Brazilian Guinea Pig, "Preá" }\end{array}$ & Inflammation & Terrestrial \\
\hline $\begin{array}{l}\text { Galea spixii (Wagler, 1831), Spix’s } \\
\text { Yellow-Toothed Cavy }\end{array}$ & Inflammation & Terrestrial \\
\hline $\begin{array}{l}\text { Kerodon rupestris (Wied-Neuwied, } \\
\text { 1820), Rock Cavy, "Mocó" }\end{array}$ & Constipation & Terrestrial \\
\hline \multicolumn{3}{|l|}{ Cebidae } \\
\hline $\begin{array}{l}\text { Alouatta belzebul (Linnaeus, 1766), } \\
\text { red-handed howler monkey, "guariba," } \\
\text { "macaco" }\end{array}$ & Whooping cough, sore throat, asthma & Terrestrial \\
\hline
\end{tabular}


TABle 1: Continued.

\begin{tabular}{|c|c|c|}
\hline Taxa & Health conditions treated & Habitat \\
\hline $\begin{array}{l}\text { Alouattanigerrima Lönnberg, 1941, } \\
\text { Black Howler Monkey }\end{array}$ & Whooping cough, inflammation & Terrestrial \\
\hline $\begin{array}{l}\text { Alouatta macconnelli (Linnaeus, } \\
\text { 1766), red howler monkey, "guariba } \\
\text { vermelho" }\end{array}$ & $\begin{array}{l}\text { Whooping cough, inflammation, accelerating } \\
\text { parturition }\end{array}$ & Terrestrial \\
\hline $\begin{array}{l}\text { Sapajus apella (Linnaeus, 1758), } \\
\text { brow capuchin, "capuchin," "macaco," } \\
\text { "macaco-prego" }\end{array}$ & Insect sting & Terrestrial \\
\hline \multicolumn{3}{|l|}{ Cervidae } \\
\hline $\begin{array}{l}\text { Blastocerus dichotomus (Illiger, 1815), } \\
\text { marsh deer, "cervo-do-pantanal" }\end{array}$ & Diarrhoea, vomit & Terrestrial \\
\hline $\begin{array}{l}\text { Mazama americana (Erxleben, } \\
\text { 1777), red brocket, "veado gaedo" }\end{array}$ & Stroke & Terrestrial \\
\hline $\begin{array}{l}\text { Mazama simplicicornis (Illinger, } \\
\text { 1811) }\end{array}$ & Diarrhoea, verminosis, evil eye & Terrestrial \\
\hline $\begin{array}{l}\text { Mazama gouazoupira (G. Fischer, } \\
\text { 1814), grey brocket, "veado-catingueiro" }\end{array}$ & $\begin{array}{l}\text { Asthma, edema, rheumatism, snake bite, } \\
\text { thrombosis, assisting children who take longer } \\
\text { than usual to start walking, toothache, wounds, } \\
\text { sprains }\end{array}$ & Terrestrial \\
\hline $\begin{array}{l}\text { Ozotocerus bezoarticus (Linnaeus, } \\
\text { 1758), Pampas Deer, veado campineiro }\end{array}$ & Diarrhoea, verminosis, evil eye & Terrestrial \\
\hline \multicolumn{3}{|l|}{ Dasypodidae } \\
\hline $\begin{array}{l}\text { Dasypus novemcinctus (Linnaeus, } \\
\text { 1758), nine-banded armadillo, "tatu } \\
\text { galinha" }\end{array}$ & $\begin{array}{l}\text { Thrombosis, insects bite, scorpions bite, edema, } \\
\text { asthma, deafness, earache, evil eye }\end{array}$ & Terrestrial \\
\hline $\begin{array}{l}\text { Euphractus sexcinctus (Linnaeus, } \\
\text { 1758), six-banded armadillo "tatu peba" }\end{array}$ & $\begin{array}{l}\text { Wounds, earache, evil eye, asthma, sore throat, } \\
\text { pneumonia, sinusitis, deafness, coarse throat }\end{array}$ & Terrestrial \\
\hline $\begin{array}{l}\text { Tolypeutes tricinctus (Linnaeus, } \\
\text { 1758), Brazilian three-banded armadillo, } \\
\text { "tatu-bola" }\end{array}$ & Thrombosis, rheumatism & Terrestrial \\
\hline $\begin{array}{l}\text { Priodontes maximus (Kerr, 1792), } \\
\text { giant armadillo, tatu-canastra }\end{array}$ & Snake bite & Terrestrial \\
\hline \multicolumn{3}{|l|}{ Dasyproctidae } \\
\hline $\begin{array}{l}\text { Dasyprocta prymnolopha Wagler, } \\
\text { 1831, black-rumped agouti, "Cutia” }\end{array}$ & Asthma, thrombosis, earache & Terrestrial \\
\hline \multicolumn{3}{|l|}{ Delphinidae } \\
\hline $\begin{array}{l}\text { Sotalia fluviatilis Gervais \& Deville } \\
\text { (1853), grey dolphin, grey river dolphin, } \\
\text { "boto" }\end{array}$ & $\begin{array}{l}\text { Asthma, headache, rheumatism, hernia, womb } \\
\text { disorders, sore throat, injuries caused by the } \\
\text { spines of the "arraia," swelling, haemorrhoids } \\
\text { inflammation, wounds, earache, erysipelas, } \\
\text { athlete's foot, tumour, cancer }\end{array}$ & Freshwater \\
\hline $\begin{array}{l}\text { Sotaliaguianensis (P. J. Van Bénéden, } \\
\text { 1864), Guianan River Dolphin, "boto" }\end{array}$ & $\begin{array}{l}\text { Asthma, headache, rheumatism, hernia, womb } \\
\text { disorders, sore throat, injuries caused by the } \\
\text { spines of the "arraia," swelling, haemorrhoids } \\
\text { inflammation, wounds, earache, erysipelas, } \\
\text { athlete's foot, tumour, cancer }\end{array}$ & Marine \\
\hline \multicolumn{3}{|l|}{ Didelphidae } \\
\hline $\begin{array}{l}\text { Didelphis albiventris (Lund, 1840), } \\
\text { White-Eared Opossum, "timbú" }\end{array}$ & Boils & Terrestrial \\
\hline $\begin{array}{l}\text { Didelphis marsupialis (Linnaeus, } \\
\text { 1758), Black-Eared Opossum, "mucura," } \\
\text { "gambá," "saruê" }\end{array}$ & $\begin{array}{l}\text { Acne, wounds, bronchitis, joint pain, stomach } \\
\text { ache, rheumatism, diarrhoea, inflammation, } \\
\text { erysipelas, pain in gestation, asthma, headache, } \\
\text { toothache, earache, sore throat }\end{array}$ & Terrestrial \\
\hline
\end{tabular}


TABle 1: Continued.

\begin{tabular}{|c|c|c|}
\hline Taxa & Health conditions treated & Habitat \\
\hline \multicolumn{3}{|l|}{ Echimyidae } \\
\hline $\begin{array}{l}\text { Thrichomys laurentius Thomas, 1904, } \\
\text { "punaré" }\end{array}$ & Diarrhoea & Terrestrial \\
\hline \multicolumn{3}{|l|}{ Erethizontidae } \\
\hline $\begin{array}{l}\text { Coendou prehensilis (Linnaeus, } \\
\text { 1758), Brazilian porcupine, "coandú," } \\
\text { "porco espinho" }\end{array}$ & $\begin{array}{l}\text { Bronchitis, thrombosis, epilepsy, } \\
\text { stroke, abscesses, conjunctivitis, } \\
\text { asthma }\end{array}$ & Terrestrial \\
\hline \multicolumn{3}{|l|}{ Hydrochaeridae } \\
\hline $\begin{array}{l}\text { Hydrochaeris hydrochaeris } \\
\text { (Linnaeus, 1766), capybara, "capibara," } \\
\text { "capivara" }\end{array}$ & $\begin{array}{l}\text { Thrombosis, conjunctivitis, } \\
\text { venereal disease, rheumatism, } \\
\text { earache, strengthen bones, liver } \\
\text { pain, bronchitis, asthma, } \\
\text { wounds, erysipelas, cough }\end{array}$ & Terrestrial \\
\hline \multicolumn{3}{|l|}{ Iniidae } \\
\hline $\begin{array}{l}\text { Inia geoffrensis (Blainville, 1817), } \\
\text { Amazon river dolphin, "boto rosa" }\end{array}$ & $\begin{array}{l}\text { Asthma, headache, rheumatism, } \\
\text { hernia, womb disorders, sore } \\
\text { throat, injuries caused by the } \\
\text { spines of the "arraia," swelling, } \\
\text { haemorrhoids inflammation, } \\
\text { wounds, earache, erysipelas, } \\
\text { athlete's foot, tumour, cancer }\end{array}$ & Freshwater \\
\hline \multicolumn{3}{|l|}{ Leporidae } \\
\hline $\begin{array}{l}\text { Sylvilagus brasiliensis (Linnaeus, } \\
\text { 1758), forest rabbit, tapeti, "coelho," } \\
\text { "coelho-do-mato" }\end{array}$ & $\begin{array}{l}\text { Thrombosis, conjunctivitis, boils, } \\
\text { burns }\end{array}$ & Terrestrial \\
\hline \multicolumn{3}{|l|}{ Mustelidae } \\
\hline $\begin{array}{l}\text { Conepatus semistriatus (Boddaert, } \\
\text { 1785), striped hog-nosed skunk, } \\
\text { "cangambá," "gambambá," tacaca }\end{array}$ & Rheumatism & Terrestrial \\
\hline $\begin{array}{l}\text { Lontra longicaudis (Olfers, 1818), } \\
\text { Neotropical Otter, "Lontra" }\end{array}$ & Thrombosis & Terrestrial \\
\hline \multicolumn{3}{|l|}{ Myrmecophagidae } \\
\hline $\begin{array}{l}\text { Myrmecophaga tridactyla Linnaeus, } \\
\text { 1758, giant anteater, "tamanduá-bandeira" }\end{array}$ & Thrombosis, stroke & Terrestrial \\
\hline $\begin{array}{l}\text { Myrmecophaga tetradactyla } \\
\text { (Linnaeus, 1758), collared anteater, } \\
\text { "tamanduá" }\end{array}$ & Edema, thrombosis & Terrestrial \\
\hline \multicolumn{3}{|l|}{ Procyonidae } \\
\hline $\begin{array}{l}\text { Nasua nasua (Linnaeus, 1766), South } \\
\text { American coati, "coati," "quati” }\end{array}$ & $\begin{array}{l}\text { Sexual impotence, wounds, skin } \\
\text { burns, snake bites, backache }\end{array}$ & Terrestrial \\
\hline $\begin{array}{l}\text { Procyon cancrivorus (G. [Baron] } \\
\text { Cuvier, 1798), crab-eating raccoon, } \\
\text { "guaxinim" }\end{array}$ & $\begin{array}{l}\text { Rheumatism, epilepsy, } \\
\text { thrombosis, snake bite }\end{array}$ & Terrestrial \\
\hline \multicolumn{3}{|l|}{ Tapiridae } \\
\hline $\begin{array}{l}\text { Tapirus terrestris (Linnaeus, 1758), } \\
\text { Brazilian tapir, "anta” }\end{array}$ & $\begin{array}{l}\text { Rheumatism, arthrosis, } \\
\text { osteoporosis, bursitis, muscular } \\
\text { pain, asthma, tonsillitis }\end{array}$ & Terrestrial \\
\hline \multicolumn{3}{|l|}{ Tayassuidae } \\
\hline $\begin{array}{l}\text { Pecari tajacu Linnaeus } 1758 \text {, collared } \\
\text { peccary, "porco-do-mato," "caititu" }\end{array}$ & Thrombosis, bronchitis, stroke & Terrestrial \\
\hline
\end{tabular}


TABLE 1: Continued.

\begin{tabular}{|c|c|c|}
\hline Taxa & Health conditions treated & Habitat \\
\hline $\begin{array}{l}\text { Tayassu pecari (Link, 1795), } \\
\text { white-lipped peccary "porco-do-mato," } \\
\text { "queixada" }\end{array}$ & Thrombosis, stroke & Terrestrial \\
\hline \multicolumn{3}{|l|}{ Trichechidae } \\
\hline $\begin{array}{l}\text { Trichechus inunguis (Natterer, 1883), } \\
\text { Amazonian manatee, "peixe-boi" }\end{array}$ & $\begin{array}{l}\text { Sprains, vaginal discharge, } \\
\text { injuries caused by bang, burns, } \\
\text { asthma, menstrual cramps, } \\
\text { rheumatism, sore throat, } \\
\text { wounds, muscle strain, sucking a } \\
\text { splinter out of skin or flesh, } \\
\text { tumour, backache, hernia, } \\
\text { arthrosis, luxation, menstrual } \\
\text { cramps, insects bite }\end{array}$ & Freshwater \\
\hline $\begin{array}{l}\text { Trichechus manatus (Linnaeus, } \\
\text { 1758), West Indian Manatee, "peixe-boi” }\end{array}$ & $\begin{array}{l}\text { Sprains, vaginal discharge, } \\
\text { injuries caused by bang, burns, } \\
\text { asthma, menstrual cramps, } \\
\text { rheumatism, sore throat, } \\
\text { wounds, muscle strain, sucking a } \\
\text { splinter out of skin or flesh, } \\
\text { tumour, backache hernia, } \\
\text { arthrosis, luxation, menstrual } \\
\text { cramps, insects bite }\end{array}$ & Marine \\
\hline \multicolumn{3}{|l|}{ Felidae } \\
\hline $\begin{array}{l}\text { Puma concolor (Linnaeus, 1771), } \\
\text { mountain lion, "onça" }\end{array}$ & Wounds, leishmaniosis & Terrestrial \\
\hline $\begin{array}{l}\text { Panthera onca (Linnaeus, 1758), } \\
\text { jaguar, "onça" }\end{array}$ & Wounds, leishmaniosis & Terrestrial \\
\hline $\begin{array}{l}\text { Herpailurus yagouaroundi (É. } \\
\text { Geoffroy Saint-Hilaire, 1803), } \\
\text { "gatovermelho," "gato-azul," Jaguarundi }\end{array}$ & Wounds & Terrestrial \\
\hline $\begin{array}{l}\text { Leopardus pardalis (Linnaeus, 1758), } \\
\text { ocelot, "gato-maracajá," }\end{array}$ & $\begin{array}{l}\text { Headache, sore throat, backache, } \\
\text { wounds }\end{array}$ & Terrestrial \\
\hline $\begin{array}{l}\text { Leopardus tigrinus (Schreber, 1775), } \\
\text { oncilla, "gato-mirim" }\end{array}$ & $\begin{array}{l}\text { Wounds, urinary incontinence, } \\
\text { injuries, sore throat, sucking a } \\
\text { splinter out of skin or flesh }\end{array}$ & Terrestrial \\
\hline
\end{tabular}

the trahira fish (Hoplias malabaricus; $n=23$ prescriptions) and the two manatee species recorded (Trichechus inunguis and T. manatus; $n=18$ prescriptions, each) also stand out for being indicated to the treatment of multiple illnesses.

Each species was prescribed to treat a mean of $4.4 \pm$ 0.78 (mean \pm confidence interval) health conditions. Reptiles contributed with the highest mean number of diseases treated per species, while birds and fishes comprised the groups with the lowest means (Kruskal-Wallis test: $H=53.209 ; n=$ 201; $p<0.01$; Dunn's post hoc test: $p<0.01$; Figure 3). Nonetheless, species showed similar number of prescriptions according to habitat type $\left(F_{4,199}=1.36 ; p=0.247\right)$.

Prescriptions of edible medicinal vertebrates were generalised in 20 disease categories, according to ICD-10. From those, "symptoms, signs, and abnormal clinical and laboratory findings" were the most recorded category in terms of therapeutic quotes recorded, followed by "infectious and parasitic diseases" and "injuries, poisoning, and other consequences of external causes" (Table 2).

With regard to the number of species associated with ICD-10 categories, most animals were prescribed for treating problems associated with the "musculoskeletal system and connective tissue" and the "respiratory system" (each: $n=80$ species; 39.2\%), "injuries, poisoning, and other consequences of external causes" (67 species, 32.8\%), and "symptoms, signs, and abnormal clinical and laboratory findings" (58 species, 28.4\%) (Table 2).

Despite most medicinal vertebrates provide raw materials for remedies, medicinal products often have magicalreligious purposes, particularly for the prevention of diseases of spiritual cause (e.g., evil eye); they were also used as amulets to prevent diseases (e.g., amulet used as a protection against snake bite). It is worth noting that many animals involved in poisoning accidents, such as stingrays and snakes, 
TABle 2: Medicinal uses of edible fishes and game species in Brazil. Health condition categories follow the International Statistical Classification of Diseases and Related Health Problems (ICD-10 Version: 2016). $N$ = total number of conditions treated in each category.

Ascites; chest pain; cough; cracks in the sole of the feet; edema (also quoted as edema in the legs); fatigue; fever; headache; hoarseness; inflammation; jaundice; lack of appetite (also quoted as lack of

Symptoms, signs, and abnormal clinical and laboratory findings, not elsewhere classified ( $n=58$ species)

Certain infectious and parasitic diseases ( $n=40$ species $)$

Injury, poisoning, and certain other consequences of external causes ( $n=67$ species $)$

Diseases of the digestive system ( $n=40$ species)

Diseases of the musculoskeletal system and connective tissue

( $n=80$ species)

Diseases of the respiratory system

( $n=80$ species)

Diseases of the skin and subcutaneous tissue $(n=21$ species $)$

Diseases of the genitourinary system ( $n=19$ species)

Pregnancy, childbirth, and the puerperium ( $n=12$ species)

Diseases of the eye and adnexa

( $n=13$ species)

External causes of morbidity and mortality

( $n=25$ species $)$

Undefined

( $n=11$ species)

Diseases of the blood and blood-forming organs and certain disorders involving the immune mechanism

( $n=12$ species)

Diseases of the circulatory system

( $n=42$ species)

Diseases of the nervous system

( $n=5$ species)

Mental and behavioural disorders ( $n=13$ species $)$ appetite in children); numbness; pain (also quoted as pain in the body; pain in the breast; pain in the legs; to reduce pain); shortness of breath; swelling; assisting children who take longer than usual to start walking; vomit.

Abscesses; athlete's foot; diphtheria; erysipelas; herpes zoster;

infection; leishmaniosis; leprosy; mumps; mycosis; schistosomiasis; syphilis; tetanus; tuberculosis; venereal disease; verminosis; warts; whooping cough.

Bruises; burns (also quoted as burns in the skin); chilblains; injuries caused by bang; injuries caused by the animal itself; injuries caused by the spines of fishes (also quoted as injuries caused by the spines of rays); intoxication from poisonous animals; pain relief in injuries caused by the species' sting; pain relief in injuries caused by snake bites; pain relief in injuries caused by sting of insects; scratch; assisting in removing spines or other sharp structures from the skin (also quoted as to suck a splinter out of skin or flesh); wounds.

Appendicitis; constipation; dental caries; diarrhoea; gastritis; haemorrhoids; hernia (also quoted as umbilical hernia); indigestion; irritation when milk teeth are erupting; liver pain; stomach ache; stomach disorders; toothache.

Arthritis; arthrosis; backache; bursitis; luxation; muscle strain; muscular pain; neck strain; osteoporosis; pain in joint; rheumatism; sprains; helping to strengthen bones.

Asthma; bronchitis; catarrh; coarse throat; cold; flu; lung disease; pneumonia; respiratory diseases; sinusitis; sore throat; tonsillitis.

Acne; blackhead; boils; calluses; dermatitis; itching; paronychia; skin diseases; skin spots; skin thorns and wounds; wrinkles.

Menstrual cramps; nephritis; prostate problems; renal failure; urinary disorders; urinary incontinence; urinary infection; discharge (also quoted as vaginal discharge); womb disorders.

Disorders after parturition (to accelerate recovery after parturition);

haemorrhage after delivery; nausea during pregnancy; pain in gestation; helping to accelerate parturition; helping to avoid swelling of the breast feeding; helping to induce abortion; helping to prevent abortion; wound in the breast caused by suckling.

Cataracts; conjunctivitis; eye pains; ophthalmological problems (also quoted as eye problems); leucoma; pterygium; stye.

Dog bite; insect sting; scorpions sting; snake bite; helping to stop the sensation of getting thirsty.

Amulet; amulet used as a protection against snake bite; evil eye; helping to remove wrath.

Anaemia; bleeding (also quoted as wounds bleeding); haemorrhage.

Stroke; thrombosis; varicocele.

Epilepsy; insomnia; paralysis of arms and legs.

Alcoholism; sexual impotence; helping to stay awake. 
TABLE 2: Continued.

\begin{tabular}{lc}
\hline ICD 10 & Indication of use and therapeutic properties \\
\hline $\begin{array}{l}\text { Endocrine, nutritional, and metabolic diseases } \\
(n=7 \text { species })\end{array}$ & Diabetes, goitre; helping to control cholesterol level. \\
$\begin{array}{l}\text { Neoplasms } \\
(n=18 \text { species })\end{array}$ & Breast cancer; cancer (also quoted as tumour). \\
$\begin{array}{l}\text { Diseases of the ear and mastoid process } \\
(n=40 \text { species })\end{array}$ & Deafness; earache. \\
$\begin{array}{l}\text { Certain conditions originating in the perinatal } \\
\text { period } \\
(n=1 \text { species })\end{array}$ & Healing of umbilical cord of newborn baby. \\
\hline
\end{tabular}

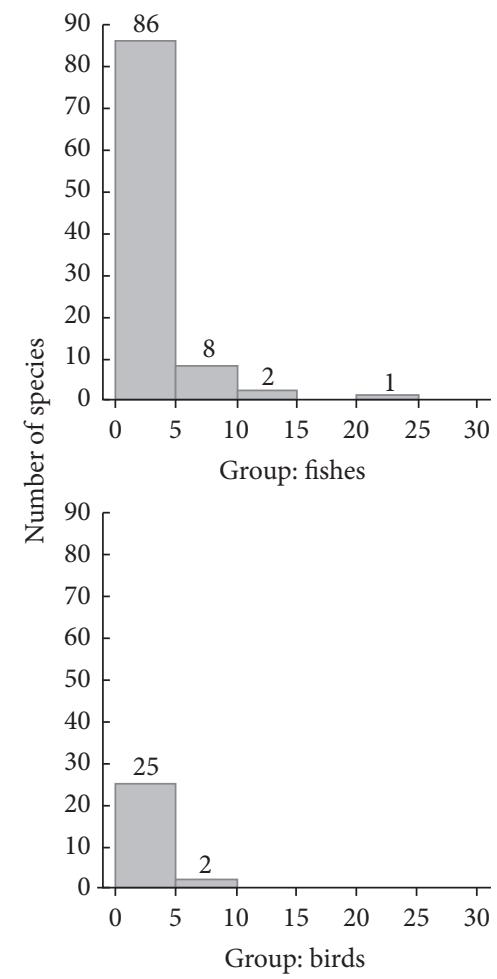

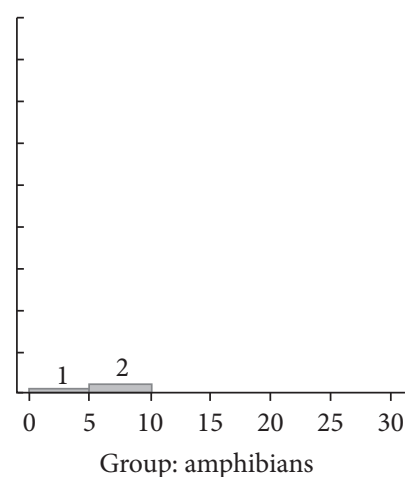
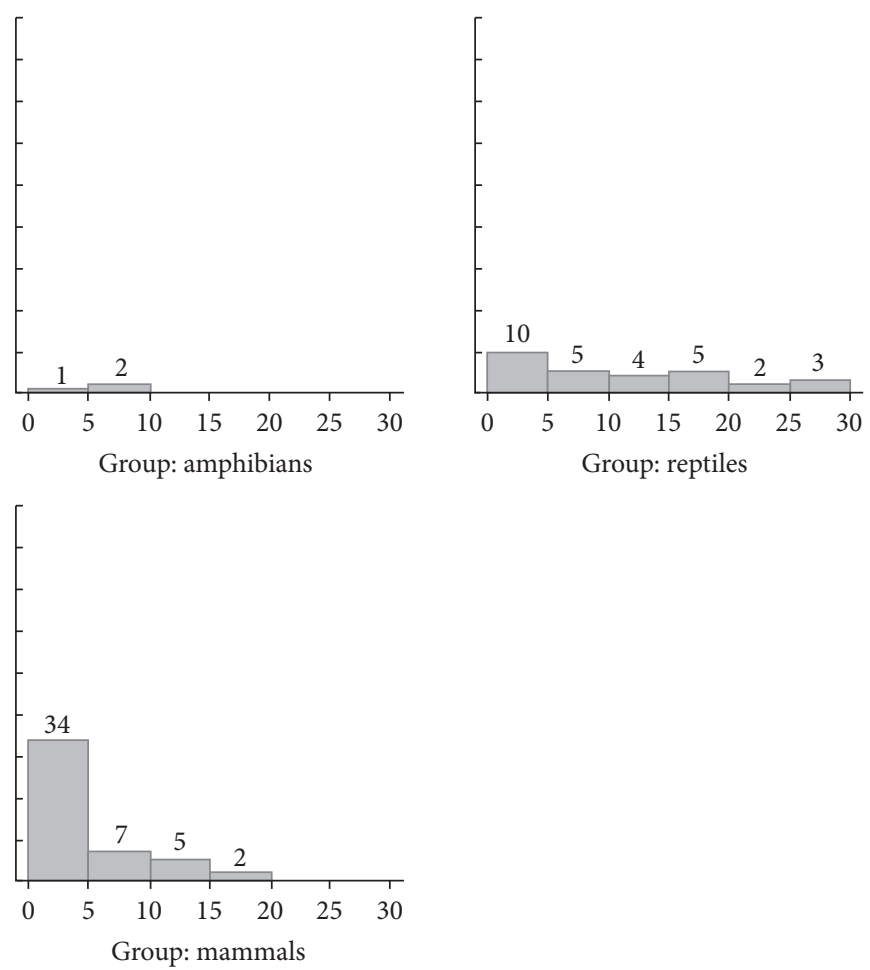

Number of treated health conditions

FIGURE 2: Number of health conditions treated by each taxonomic group of edible vertebrates used in traditional medicines in Brazil, according to their taxonomic group. Numbers above bars: number of animal species analysed.

are also used in folk medicine, particularly to treat injuries caused by themselves (see Table 1).

Fishes and birds appear to have most similar use according to ICD-10 categories (Jaccard index: 94.4), as well as reptiles and mammals (Jaccard index: 90.0), resulting in two distinct clusters (Figure 4(a)). When considering resemblance between the disease categories recorded and animals' habitat types, two distinct clusters were also formed (terrestrial, freshwater, coastal, and marine; costal and marine/freshwater and semiaquatic) (Figure 4(b)), thus reflecting highest similarities between continental habitats (terrestrial and freshwater; Jaccard index: 90.0).

With regard to species conservation status, 160 animals figure in at least one of the three red lists assessed (see
Table 1). In the ICUN red list, 33 species (mainly fishes and mammals) are classified into threatened categories, mostly as vulnerable (VU; $n=27$ ) ones. Endangered (EN) and critically endangered (CR) species comprised six fishes and reptiles, namely, Narcine bancroftii and Pristis pectinata (CR) and Sphyrna lewini, S. mokarran, Chelonia mydas, and Eretmochelys imbricata (EN). In Brazilian red lists, most threatened animals are also considered VU $(n=22)$; EN species $(n=9)$ comprise mainly fishes and mammals; and CR ones $(n=8)$ comprise mainly fishes and marine reptiles. In CITES, 58 species are listed, especially in its Appendix II ( $n=37$ ), mammals and reptiles being the most expressive groups. 


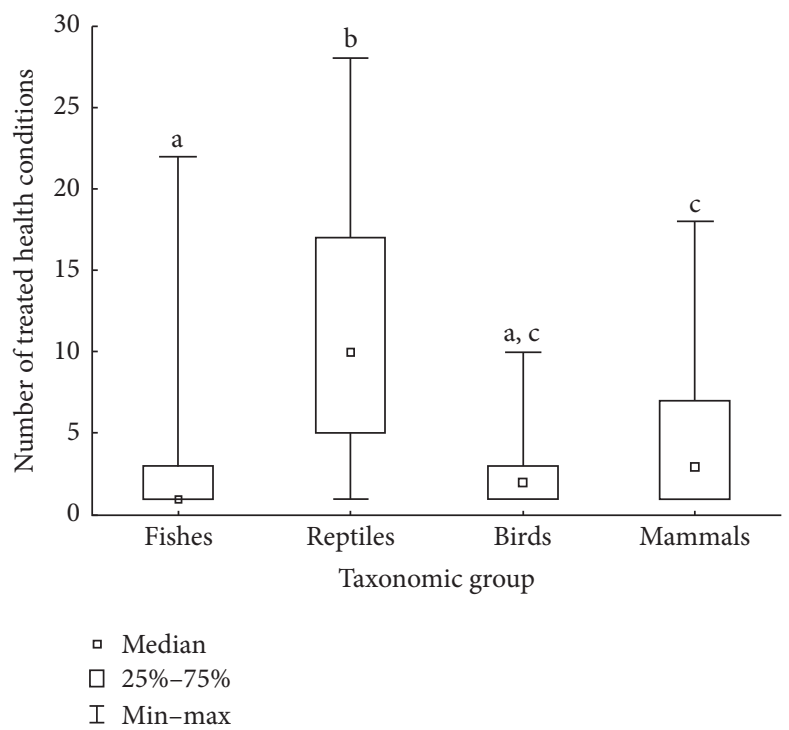

FIGURE 3: Number of health conditions treated per species, according to taxonomic group. Letters indicate the results of Dunn's post hoc test: shared letters mean no statistical difference between groups $(p>0.05)$.

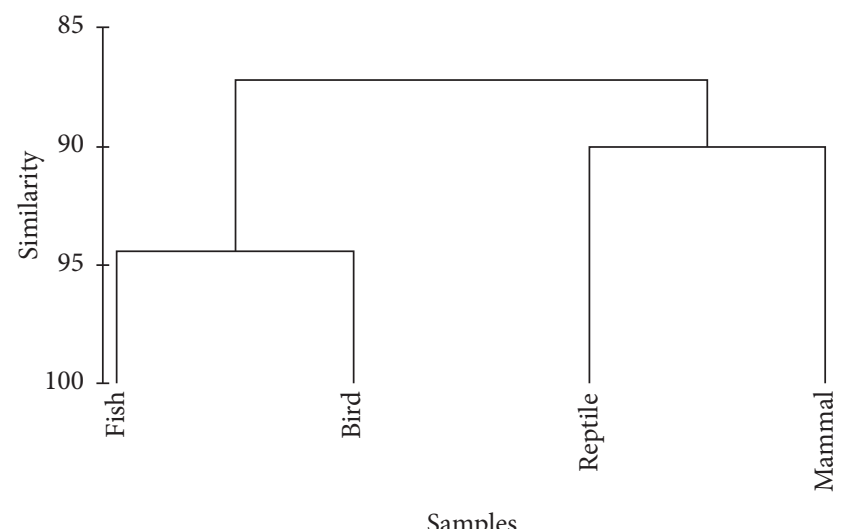

(a)

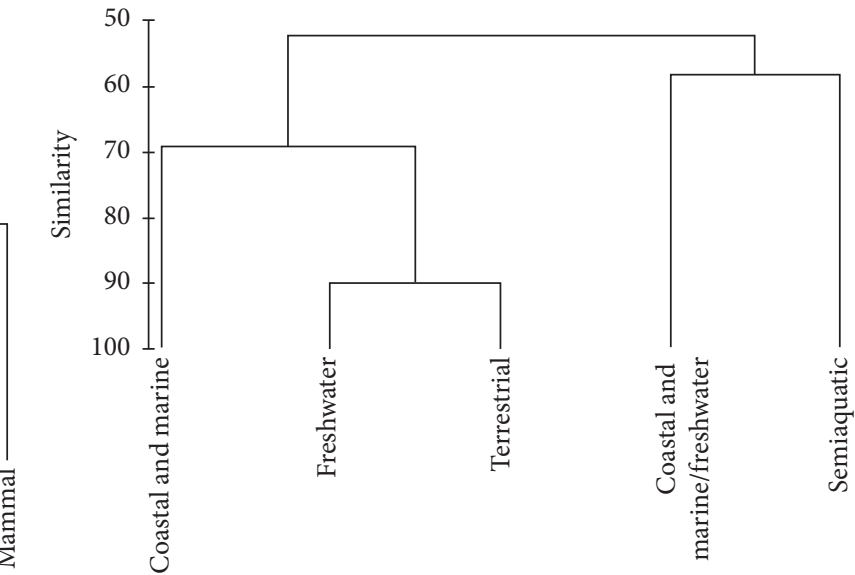

Samples

(b)

FIGURE 4: Dendogram showing taxonomic group (a) and habitat type (b) similarities with ICD-10 disease categories.

\section{Discussion}

The high number of vertebrates used as medicine is not surprising given the important role played by wildlife as a source of medicines in different traditional medicine systems $[8,10,23,24]$. The predominance of fishes and mammals in the Brazilian Traditional Medicine confirms our expectations, given that those groups comprise major targets in Brazil [25-28]. Although these two taxa have been primarily harvested for alimentary purposes, they generate a series of the inedible parts [such as bone, skin, tail, feather, liver, and bile ("fel")], rattle (from rattlesnakes), spine, scale, penis, carapace, beak, teeth, head, nails, and horn that can be used in popular medicines. According to Moura and Marques [29] the use of leftover/secondary products derived from the fauna seems to be one of the most conspicuous features on the Brazilian popular zootherapy.

Zootherapeutic products, however, do not include inedible parts solely: flesh, eggs, and viscera are among some animal products used for both medicinal and alimentary purposes $[1,12,13,30,31]$. This corroborates the assumption that the consumption of wild vertebrates meat is often related to the purported medicinal or cultural benefits derived from the animal parts [32-35]. In a recent review study, Alves et al. [15] pointed out that at least 354 wild animal species are used in Brazilian Traditional Medicine, of which 157 are also used as food, evidencing that a close connection between eating and healing is common in Brazilian zootherapy. This is in line with several studies in ethnobiology and ethnopharmacology that have observed how difficult the clear separation between 
medicines and foods can be [36-38] and this situation includes plants and animals, essential items for the preparation of traditional medicine.

Whether for food or medicinal purposes, the consumption of wild animals can lead to the transmission of various human diseases [39]. Van Vliet et al. [40] highlighted that the consumption of bushmeat for either purpose may lead to human infection by several zoonotic pathogens. Armadillos, for example, are widely used in folk medicine and are a natural reservoir of etiological agents of several zoonotic diseases that affect humans, such as leprosy, trichinosis, coccidioidomycosis or Valley Fever, Chagas disease, and typhus [41]. Therefore, it is essential that traditional drug therapies are submitted to an appropriate benefit/risk analysis [39].

It was found that several medicinal vertebrates used in the Brazilian Traditional Medicine have multiple therapeutic indications. The possibility of using various remedies for the same ailment is popular because it allows adapting to the availability of the animals. The fact that some medicinal animals are being used for the same purpose suggests that different species can share similar medicinal properties and might indicate the pharmacological effectiveness of those zootherapeutic remedies [8].

Multiple medicinal uses become even more evident when considering reptiles, as this group comprises one of the most important animal resources related to the medicine history [42] and is widely used in the most important traditional pharmacopeias worldwide [35]. Indeed, the use in traditional medicines is the human practice that involves the highest diversity of reptile species in Brazil [17], some of which play important roles in traditional medicines, such as the "teju" (Tupinambis teguixin) and the boa snake (Boa constrictor), which are one of the most used medicinal animals in Brazil $[42,43]$. Curiously, there is a general aversion to consuming some reptile groups, such as snakes and lizards, in the country. Nonetheless, this fact does not impair the use of these animals as medicines, as it is mainly associated with popular beliefs known as "simpatias," which, in most of the cases, state that "a person receiving a given treatment cannot know what that he/she is taking, otherwise the effect ceases" [18]. Hence, this fact seems to favour the high use of reptile species, despite widespread aversion to those animals.

On the other hand, despite presenting the highest diversity of medicinal species, fishes were recommended to treat a comparatively low number of health conditions. This may be related to the fact that most parts of a fish are consumed as food; thus fewer products are left to be used in medicinal practices. Similarly, when considering major hunted taxa in Brazil, that is, mammals and birds [25, 26, 44], most species are also mostly consumed as food. However, the inedible parts generate "leftovers" (e.g., skin, tail, spine, scale, teeth, nails, and horn) which are among the main products used in traditional medicine. Indeed, according to Moura and Marques [29], the zootherapeutic use of the fauna is mainly based on derived leftovers/secondary products. Those authors also emphasise that, from the ecological theory point of view, the use of leftovers could be justified as an attempt to leverage the resources obtained from ecosystems which are inappropriate for alimentary consumption due to the mechanical difficulty of ingesting these parts, such as horns, feather, and scales. Therefore, one can expect that the diversity of leftovers provided by a species may support the potential to treat multiple diseases.

Animals from continental habitats (i.e., terrestrial and freshwater) were found to treat similar disease categories; the same could be found within coastal and marine animals. This may be related to the local distribution of the diseases treated, thus leading people to use local resources in the traditional medicine of each region. For instance, in coastal areas, the occurrence of diseases classified into the category "external causes of morbidity and mortality" is very common, due to sting/poisoning accidents caused by fishes (e.g., stingrays, catfish, and toadfish), which are often treated by zootherapeutic products derived from the animals that caused the lesions [45-48].

Natural resources play an essential role in health care in traditional medical systems, as well as in bioprospecting for new drugs $[49,50]$, and the interest in animal-based products has raised $[49,51,52]$. Hence, despite the available information on the chemical components and actions of some of these products, studies on fauna traditional uses still are potentially very important to shed light on several aspects of their therapeutic applications [53].

The comprehension of the multiplicity and trends in therapeutic uses of several vertebrate species is of particular interest from a conservation perspective, as threatened animals, such as those recorded in this and other studies [30] could be replaced by nonthreatened species with similar properties. However, it is important to highlight that the use of animals for both food and medicinal purposes may impose higher pressure on those species under overexploitation conditions. For instance, if the animal is solely sought for medicinal purposes, it can lead the hunter/fisher to use selective capture techniques or even release nontargeted species. On the other hand, if an animal is captured for feeding reasons and is not the main target of the hunting or fishery (e.g., due to size), it can be kept by the hunter/fisher due to some medicinal property. Hence, understanding such complex interactions and trends in the use of fauna for nutritional and medicinal purposes evidences the important role that ethnobiological and ethnopharmacological studies may play in crucial discussion on the trade-offs between animal harvesting and its sustainability towards better regulation of those practices.

\section{Conclusion}

Wild edible vertebrates, particularly those inhabiting aquatic environments, are used to treat a wide range of health conditions in Brazil, with reptiles consisting of the most versatile group in multiple disease prescriptions. Moreover, a trend in prescriptions was found according to animals' habitats, as disease categories were similar within continental and within coastal and marine habitats. Several consumed species are under threat, leading to a raise in conservation concerns, particularly due to the dual function (as food and medicines) those species present. 


\section{Conflicts of Interest}

The authors declare that they have no conflicts of interest.

\section{References}

[1] R. R. N. Alves and I. L. Rosa, Animals in Traditional Folk Medicine: Implications for Conservation, Springer, Berlin, Germany, 2013.

[2] F. Alakbarli, Medical Manuscripts of Azerbaijan, Heydar Aliyev Foundation, Baku, Azerbaijan, 2006.

[3] A. Moquin-Tandon, Elements of Medical Zoology, Baillière, 1861.

[4] A. Scarpa, "Pre-scientific medicines: their extent and value," Social Science and Medicine. Part A Medical Psychology and Medical, vol. 15, no. 3, pp. 317-326, 1981.

[5] J. Stephenson, Medical Zoology, And Mineralogy; Or, Illustrations and Descriptions of the Animals and Minerals Employed in Medicine, and of the Preparations Derived from Them: Including Also an Account of Animal and Mineral Poisons, John Wilson, London, UK, 1832.

[6] E. Unnikrishnan, Materia Medica of the Local Health Traditions of Payyannur, Centre for Development Studies, 2004.

[7] K. P. Groark, "To warm the blood, to warm the flesh: the role of the steambath in Highland Maya (Tzeltal-Tzotzil) ethnomedicine," Journal of Latin American Lore, vol. 20, no. 1, pp. 3-96, 1997.

[8] R. R. N. Alves and H. N. Alves, "The faunal drugstore: animalbased remedies used in traditional medicines in Latin America," Journal of Ethnobiology and Ethnomedicine, vol. 7, article 9, 2011.

[9] G. J. Martínez, "Use of fauna in the traditional medicine of native Toba (qom) from the Argentine Gran Chaco region: an ethnozoological and conservationist approach," Ethnobiology and Conservation, vol. 2, no. 2, pp. 1-43, 2013.

[10] M. M. Mahawar and D. P. Jaroli, "Traditional zootherapeutic studies in India: a review," Journal of Ethnobiology and Ethnomedicine, vol. 4, no. 1, article 17, 2008.

[11] F. B. Barros, S. A. M. Varela, H. M. Pereira, and L. Vicente, "Medicinal use of fauna by a traditional community in the Brazilian Amazonia," Journal of Ethnobiology and Ethnomedicine, vol. 8, article 37, 2012.

[12] F. S. Ferreira, H. Fernandes-Ferreira, N. A. Léo Neto, S. V. Brito, and R. R. N. Alves, "The trade of medicinal animals in Brazil: current status and perspectives," Biodiversity and Conservation, vol. 22, no. 4, pp. 839-870, 2013.

[13] F. S. Ferreira, U. P. Albuquerque, H. D. M. Coutinho, W. D. O. Almeida, and R. R. D. N. Alves, "The trade in medicinal animals in Northeastern Brazil," Evidence-Based Complementary and Alternative Medicine, vol. 2012, Article ID 126938, 20 pages, 2012.

[14] J. G. W. Marques, "A fauna medicinal dos índios Kuna de San Blas (Panamá) e a hipótese da universalidade zooterapica," in Proceedings of the Anais da 46 a Reunião Anual da SBPC, Vitória, Brazil, 1994.

[15] R. R. N. Alves, T. P. R. Oliveira, and I. L. Rosa, "Wild animals used as food medicine in Brazil," Evidence-Based Complementary and Alternative Medicine, vol. 2013, Article ID 670352, 12 pages, 2013.

[16] A. Perry, "Global survey of marine medicinals," in Proceedings of the 1st International Workshop on the Management and Culture of Marine Species Used in Traditional Medicines, M.-A. Moreau, H. J. Hall, and A. C. J. Vincent, Eds., pp. 35-43, Project Seahorse, Montreal, Canada, 2000.
[17] R. R. N. Alves, N. A. Léo Neto, G. G. Santana, W. L. S. Vieira, and W. O. Almeida, "Reptiles used for medicinal and magic religious purposes in Brazil," Applied Herpetology, vol. 6, no. 3, pp. 257274, 2009.

[18] R. R. N. Alves, I. L. Rosa, and G. G. Santana, "The role of animal-derived remedies as complementary medicine in Brazil," BioScience, vol. 57, no. 11, pp. 949-955, 2007.

[19] R. R. N. Alves, G. G. Santana, and I. L. Rosa, "The role of animalderived remedies as complementary medicine in Brazil," in Animals in Traditional Folk Medicine: Implications for Conservation, R. R. N. Alves and I. L. Rosa, Eds., pp. 289-301, Springer, Berlin, Germany, 2013.

[20] D. E. Wilson and D. M. Reeder, Mammal Species of the World, a Taxonomic and Geographic Reference, The Johns Hopkins University Press, Baltimore, Md, USA, 3rd edition, 2005.

[21] IUCN, "IUCN 2015. IUCN Red List of Threatened Species. Version 2015.2," http://www.iucnredlist.org/.

[22] CITES, “CITES species database," http://www.cites.org/eng/ resources/species.html.

[23] D. Ashwell and N. Walston, An Overview of the Use and Trade of Plants and Animals in Traditional Medicine Systems in Cambodia, TRAFFIC Southeast Asia, Greater Mekong Programme, Ha Noi, Vietnam, 1st edition, 2008.

[24] V. L. Williams, A. B. Cunningham, R. K. Bruyns, and A. C. Kemp, "Birds of a feather: quantitative assessments of the diversity and levels of threat to birds used in African traditional medicine," in Animals in Traditional Folk Medicine: Implications for Conservation, R. R. N. Alves and I. L. Rosa, Eds., pp. 383420, Springer, 2013.

[25] R. R. Barboza, S. F. Lopes, W. M. S. Souto, H. FernandesFerreira, and R. R. N. Alves, "The role of game mammals as bushmeat In the Caatinga, northeast Brazil," Ecology and Society, vol. 21, no. 2, article 2, 2016.

[26] R. S. de Melo, O. C. da Silva, A. Souto, R. R. N. Alves, and N. Schiel, "The role of mammals in local communities living in conservation areas in the Northeast of Brazil: an ethnozoological approach," Tropical Conservation Science, vol. 7, no. 3, pp. 423-439, 2014.

[27] M. S. P. Rocha, J. S. Mourão, W. M. S. Souto, R. R. D. Barboza, and R. R. N. Alves, "Uso dos recursos pesqueiros no Estuário do Rio Mamanguape, Estado da Paraíba, Brasil," Interciencia, vol. 33, no. 12, pp. 903-909, 2008.

[28] M. C. Sousa, I. M. Martins, and N. Hanazaki, "Trophic relationships between people and resources: fish consumption in an artisanal fishers neighborhood in southern brazil," Ethnobiology and Conservation, vol. 5, no. 4, article 4, 2016.

[29] F. B. P. Moura and J. G. W. Marques, "Zooterapia popular na Chapada Diamantina: uma Medicina incidental?" Ciência \& Saúde Coletiva, vol. 13, pp. 2179-2188, 2008.

[30] F. S. Ferreira, S. V. Brito, W. de Oliveira Almeida, and R. R. N. Alves, "Conservation of animals traded for medicinal purposes in Brazil: can products derived from plants or domestic animals replace products of wild animals?" Regional Environmental Change, vol. 16, no. 2, pp. 543-551, 2016.

[31] E. M. Costa-Neto, "Healing with animals in Feira de Santana City, Bahia, Brazil," Journal of Ethnopharmacology, vol. 65, no. 3, pp. 225-230, 1999.

[32] J. B. Thorbjarnarson, C. J. Lagueux, D. Bolze, M. W. Klemens, and A. B. Meylan, "Human use of turtle: a worldwide perspective," in Turtle Conservation, M. W. Klemens, Ed., pp. 33-84, Smithsonian Institution Press, London, UK, 2000. 
[33] M. W. Klemens and J. B. Thorbjarnarson, "Reptiles as a food resource," Biodiversity and Conservation, vol. 4, no. 3, pp. 281298, 1995.

[34] E. L. Bennett and J. G. Robinson, "Hunting of wildlife in tropical forests," The World Bank Environment Department Papers 76, 2000.

[35] R. R. Da Nóbrega Alves, W. L. Da Silva Vieira, and G. G. Santana, "Reptiles used in traditional folk medicine: conservation implications," Biodiversity and Conservation, vol. 17, no. 8, pp. 2037-2049, 2008.

[36] N. L. Etkin, Eating on the Wild Side: The Pharmacologic, Ecologic and Social Implications of Using Noncultigens, University of Arizona Press, 2000.

[37] V. Reyes-García, "Eating and Healing. Traditional Food as Medicine," Economic Botany, vol. 60, no. 4, pp. 389-389, 2006.

[38] A. Pieroni and A. Grazzini, "Alimenti-medicina di origine animale," in Herbs, Humans and Animals/Erbe, Uomini e Bestie, A. Pieroni, Ed., pp. 155-171, VDM, London, UK, 1999.

[39] P. A. G. M. De Smet, "Is there any danger in using traditional remedies?" Journal of Ethnopharmacology, vol. 32, no. 1-3, pp. 43-50, 1991.

[40] N. Van Vliet, J. Moreno, J. Gómez et al., "Bushmeat and human health: assessing the Evidence in tropical and sub-tropical forests," Ethnobiology and Conservation, vol. 6, pp. 1-45, 2017.

[41] E. A. Silva, P. S. Rosa, M. S. Arruda, and E. M. Rúbio, "Determination of Duffy phenotype of red blood cells in Dasypus novemcinctus and Cabassous sp," Brazilian Journal of Biology, vol. 65 , no. 3, pp. 555-557, 2005.

[42] R. R. N. Alves, W. L. S. Vieira, G. G. Santana, K. S. Vieira, and P. F. G. P. Montenegro, "Herpetofauna used in traditional folk medicine: conservation implications," in Animals in Traditional Folk Medicine: Implications for Conservation, R. R. N. Alves and I. L. Rosa, Eds., pp. 109-133, Springer, Berlin, Germany, 2013.

[43] H. Fernandes-Ferreira, S. V. Mendonça, R. L. Cruz, D. M. Borges-Nojosa, and R. R. N. Alves, "Hunting of herpetofauna in montane, coastal and dryland areas of Northeastern Brazil," Herpetological Conservation and Biology, vol. 8, no. 3, pp. 652666, 2013.

[44] G. P. Mesquita and L. N. Barreto, "Evaluation of mammals hunting in indigenous and rural localities in Eastern Brazilian Amazon," Ethnobiology and Conservation, vol. 4, no. 2015, article 2, 2015.

[45] R. R. N. Alves and I. L. Rosa, "Zootherapeutic practices among fishing communities in North and Northeast Brazil: a comparison," Journal of Ethnopharmacology, vol. 111, no. 1, pp. 82-103, 2007.

[46] A. Begossi, "Food taboos at Búzios Island (SE Brazil): their significance and relation to folk medicine," Journal of Ethnobiology, vol. 12, no. 1, pp. 117-139, 1992.

[47] E. N. Anderson, D. Pearsall, E. Hunn, and N. Turner, "Ethnozoology of caiçaras from Aventureiro, Ilha Grande," Journal of Ethnobiology, vol. 21, no. 1, pp. 107-135, 2001.

[48] A. Begossi and M. Ramires, "Fish folk medicine of Caiçara (Atlantic Coastal forest) and Caboclo (Amazon forest) communities," in Animals in Traditional Folk Medicine: Implications for Conservation, R. R. N. Alves and I. L. Rosa, Eds., pp. 91-108, Springer, Berlin, Germany, 2013.

[49] R. R. N. Alves and U. P. Albuquerque, "Animals as a source of drugs: bioprospecting and biodiversity conservation," in Animals in Traditional Folk Medicine: Implications for Conservation, R. R. N. Alves and I. L. Rosa, Eds., pp. 67-89, Springer, Berlin, Germany, 2013.
[50] J. Rose, C. L. Quave, and G. Islam, “The four-sided triangle of ethics in bioprospecting: pharmaceutical business, international politics, socio-environmental responsibility and the importance of local stakeholders," Ethnobiology and Conservation, vol. 1, no. 2012, article 3, 2012.

[51] E. Chivian, Biodiversity: Its Importance to Human Health, Center for Health and the Global Environment, 2002.

[52] A. T. Dossey, "Insects and their chemical weaponry: new potential for drug discovery," Natural Product Reports, vol. 27, no. 12, pp. 1737-1757, 2010.

[53] A. Pieroni, C. Quave, S. Nebel, and M. Heinrich, "Ethnopharmacy of the ethnic Albanians (Arbëreshë) of northern Basilicata, Italy," Fitoterapia, vol. 73, no. 3, pp. 217-241, 2002. 


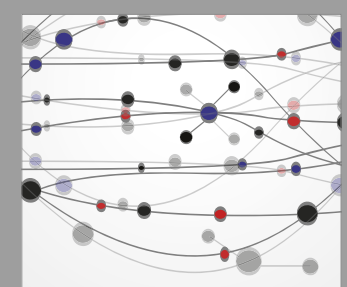

The Scientific World Journal
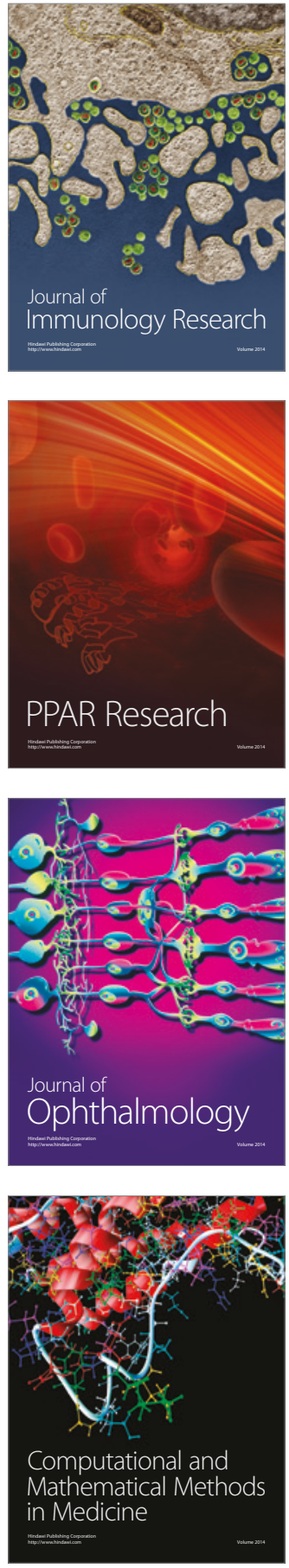

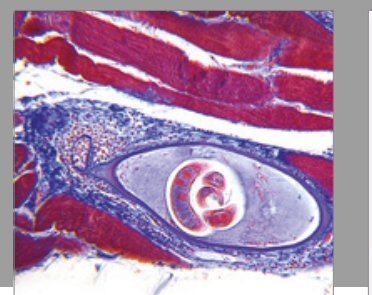

Gastroenterology Research and Practice
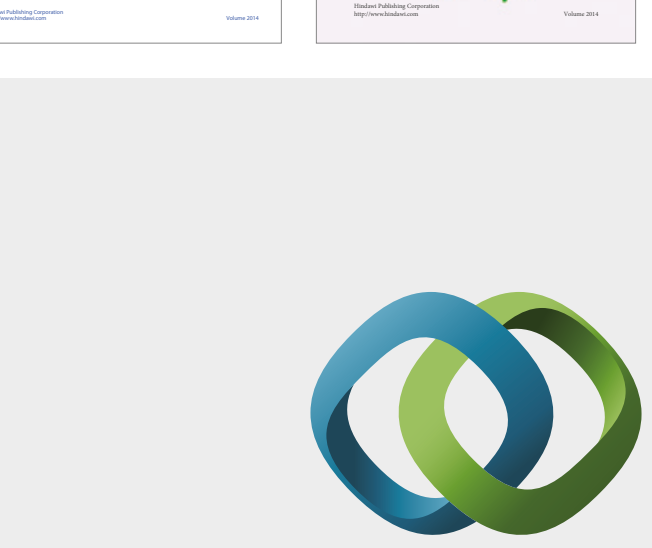

\section{Hindawi}

Submit your manuscripts at

https://www.hindawi.com
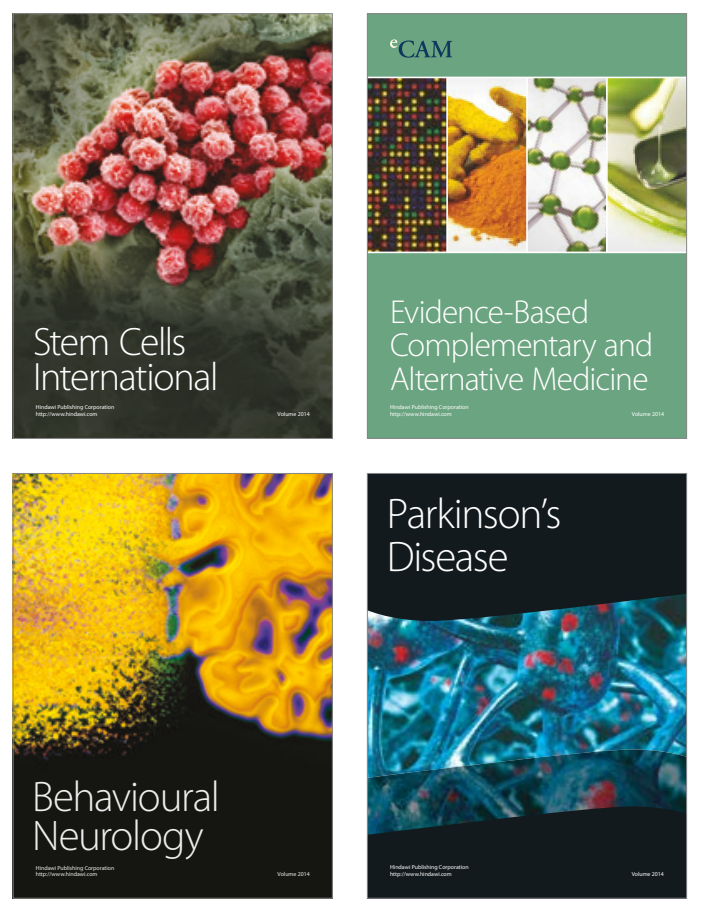
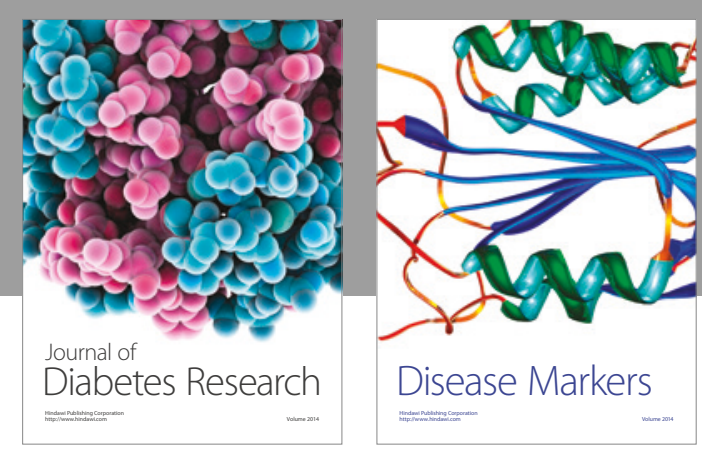

Disease Markers
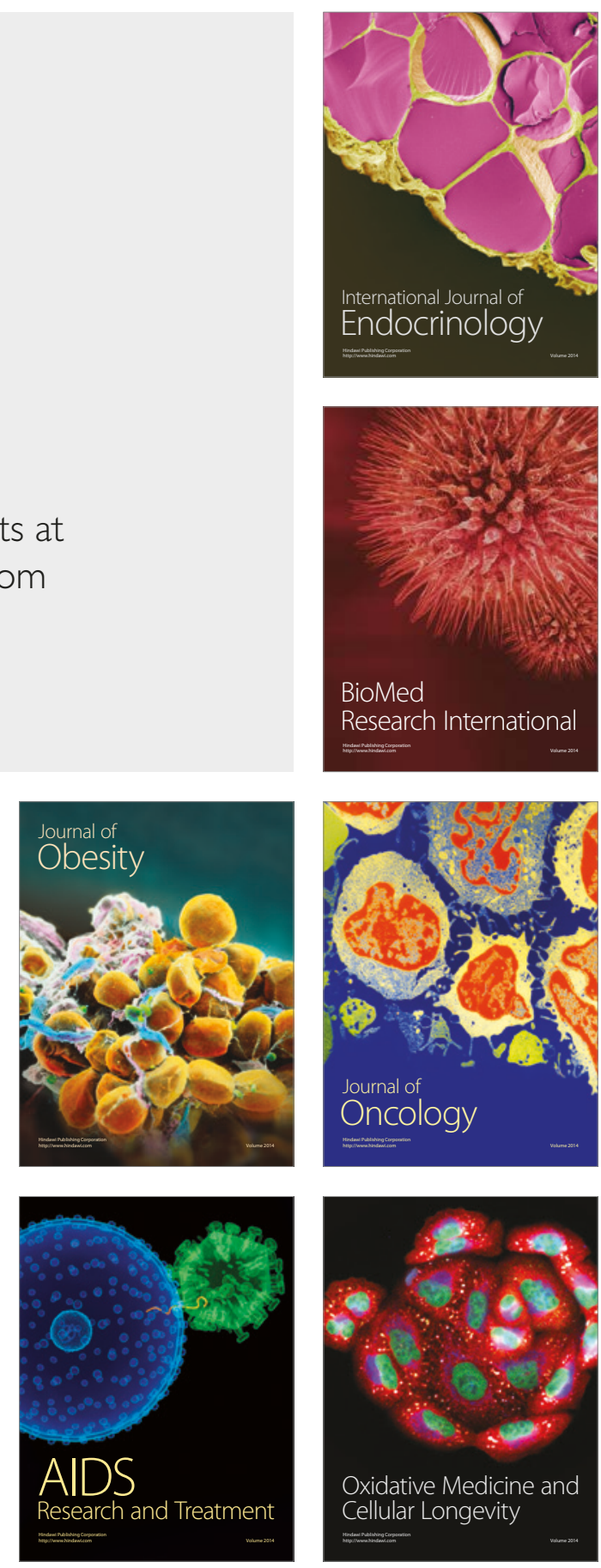